\title{
Lipid-Based Nutrient Supplementation Increases HDL Cholesterol Efflux Capacity and is Associated with Changes in the HDL Glycoproteome in Children
}

Brian V. Hong ${ }^{a}$, Chenghao Zhu ${ }^{a}$, Maurice Wong ${ }^{\mathrm{b}}$, Romina Sacchia, Christopher H. Rhodes ${ }^{\mathrm{a}}$, Jea Woo Kang ${ }^{a}$, Charles D. Arnolda, Seth Adu-Afarwuahc, Anna Lartey ${ }^{c}$, Brietta M. Oaks ${ }^{d}$, Carlito B. Lebrilla $^{\mathrm{b}}$, Kathryn G. Deweya ${ }^{\mathrm{a}}$ Angela M. Zivkovic ${ }^{\mathrm{a}^{*}}$

${ }^{a}$ Department of Nutrition and ${ }^{b}$ Chemistry, University of California, Davis, Davis, CA, USA; ${ }^{c}$ Department of Nutrition and Food Science, University of Ghana, Legon, Ghana; ${ }^{d}$ Department of Nutrition and Food Sciences, University of Rhode Island, Kingston, RI, USA

*To whom correspondence should be addressed. E-mail: amzivkovic@ucdavis.edu

Online Supporting Information: Tables S1-6, Figure S1, Method S1-2 


\begin{tabular}{|c|c|c|c|}
\hline Treatment & $\begin{array}{l}\text { Lipid Species ( } \mathrm{n}= \\
313 \text { ) }\end{array}$ & Mean (\%) & SD \\
\hline IFA & CE 16:1 & 0.1378572 & 0.0604765 \\
\hline IFA & CE 18:1 & 2.6030622 & 0.75991 \\
\hline IFA & CE 18:2 & 16.2803028 & 3.5456791 \\
\hline IFA & CE 18:3 & 0.1464146 & 0.0369396 \\
\hline IFA & CE 20:3 & 0.2330877 & 0.0856682 \\
\hline IFA & CE 20:4 & 1.8760629 & 0.6399473 \\
\hline IFA & CE 20:5 & 0.387712 & 0.2444259 \\
\hline IFA & CE 22:6 & 0.6374623 & 0.1987288 \\
\hline IFA & Ceramide $42: 2 \mathrm{~d}$ & 0.0043721 & 0.0018045 \\
\hline IFA & Cholesterol & 7.853734 & 1.2850934 \\
\hline IFA & DG 32:1 & 0.0345559 & 0.0189325 \\
\hline IFA & DG 34:1 & 0.1713322 & 0.0858138 \\
\hline IFA & DG 34:2 & 0.1330312 & 0.0666908 \\
\hline IFA & DG 34:3 & 0.0088923 & 0.0065974 \\
\hline IFA & DG 36:1 & 0.0247751 & 0.0138689 \\
\hline IFA & DG 36:2 & 0.1829097 & 0.0884051 \\
\hline IFA & DG 36:3 & 0.2134531 & 0.0900126 \\
\hline IFA & DG 36:4 A & 0.0370132 & 0.0209697 \\
\hline IFA & DG 38:5 & 0.0368875 & 0.0142032 \\
\hline IFA & DG 38:6 & 0.0246981 & 0.0115721 \\
\hline IFA & GlcCer 40:1 d & 0.0058233 & 0.0016308 \\
\hline IFA & GlcCer 42:1 d & 0.0099992 & 0.0024896 \\
\hline IFA & GlcCer 42:2 d & 0.0102188 & 0.0031231 \\
\hline IFA & Gal-Gal-Cer 42:2 d & 0.0009613 & 0.0003257 \\
\hline IFA & LPC 20:0 & 0.0017111 & 0.0010692 \\
\hline IFA & LPC 20:1 & 0.0010695 & 0.000808 \\
\hline IFA & LPC 22:4 & 0.0013012 & 0.0009683 \\
\hline IFA & LPC 22:6 & 0.0027707 & 0.0016736 \\
\hline IFA & LPC 16:0 o & 0.0044347 & 0.0043819 \\
\hline IFA & LPC $16: 0 p$ & 0.0030814 & 0.0023026 \\
\hline IFA & LPC 16:0 & 0.0521558 & 0.0339188 \\
\hline IFA & LPC 18:0 & 0.0933325 & 0.0812941 \\
\hline IFA & LPC 18:2 & 0.004914 & 0.002191 \\
\hline IFA & LPC $20: 3$ & 0.0008577 & 0.0005119 \\
\hline IFA & LPC 20:4 & 0.0038756 & 0.0017815 \\
\hline IFA & LPC 20:5 & 0.0005545 & 0.0004261 \\
\hline IFA & PC 25:0 & 0.0056509 & 0.003086 \\
\hline IFA & PC $37: 3$ & 0.0070631 & 0.0020255 \\
\hline IFA & PC 39:6 & 0.0186046 & 0.0098897 \\
\hline IFA & PC 42:5 & 0.0032337 & 0.0012247 \\
\hline
\end{tabular}




\begin{tabular}{|c|c|c|c|}
\hline IFA & PC 42:6 & 0.001678 & 0.0011308 \\
\hline IFA & PC 32:0 p & 0.0331638 & 0.0085406 \\
\hline IFA & PC 36:3 p & 0.2746408 & 0.0721579 \\
\hline IFA & PC $38: 2 p$ & 0.0073635 & 0.0019078 \\
\hline IFA & PC $42: 3 p$ & 0.0074855 & 0.0017851 \\
\hline IFA & PC $34: 1$ & 0.0625206 & 0.0131247 \\
\hline IFA & PC 36:3 & 0.0763789 & 0.0168516 \\
\hline IFA & PC 36:5 & 0.631321 & 0.1060358 \\
\hline IFA & PC 38:4 & 0.035043 & 0.012812 \\
\hline IFA & PC 40:6 & 0.0257932 & 0.0092326 \\
\hline IFA & PC 28:0 & 0.0052521 & 0.0039004 \\
\hline IFA & PC 30:0 & 0.1326906 & 0.0752787 \\
\hline IFA & PC 31:0 & 0.0031081 & 0.001485 \\
\hline IFA & PC 32:0 & 0.1684637 & 0.0460285 \\
\hline IFA & PC 32:1 & 0.1883644 & 0.1053984 \\
\hline IFA & PC 33:0 & 0.0048116 & 0.0019503 \\
\hline IFA & PC 33:1 & 0.026403 & 0.0113446 \\
\hline IFA & PC $33: 2$ & 0.0258754 & 0.0093034 \\
\hline IFA & PC 34:0 & 0.0315977 & 0.0103688 \\
\hline IFA & PC 34:1.1 & 5.8217695 & 1.7982886 \\
\hline IFA & PC $34: 2$ & 12.73141 & 1.8240495 \\
\hline IFA & PC $34: 3$ & 0.02341 & 0.0157101 \\
\hline IFA & PC 35:1 & 0.0265742 & 0.0080173 \\
\hline IFA & PC $35: 2$ & 0.0621283 & 0.0299071 \\
\hline IFA & PC 35:3 & 0.0155381 & 0.0065739 \\
\hline IFA & PC 35:4 & 0.0072446 & 0.0019098 \\
\hline IFA & PC 36:1 & 0.6186497 & 0.2206464 \\
\hline IFA & PC $36: 2$ & 5.9438642 & 1.4155724 \\
\hline IFA & PC 36:3.1 & 1.0766842 & 0.3876907 \\
\hline IFA & PC 36:3.2 & 1.145882 & 0.3222141 \\
\hline IFA & PC 36:4 & 0.2463351 & 0.1274005 \\
\hline IFA & PC 36:4.1 & 0.0833027 & 0.0404342 \\
\hline IFA & PC 36:5.1 & 0.0103022 & 0.0179414 \\
\hline IFA & PC 36:6 & 0.0132486 & 0.0075397 \\
\hline IFA & PC 37:4 & 0.0189128 & 0.0105022 \\
\hline IFA & PC 37:6 & 0.0065841 & 0.0021936 \\
\hline IFA & PC 38:2 & 0.0401641 & 0.0088659 \\
\hline IFA & PC $38: 3$ & 0.3974327 & 0.1247121 \\
\hline IFA & PC 38:4.1 & 0.0863833 & 0.0339879 \\
\hline IFA & PC 38:5 & 0.6184815 & 0.167185 \\
\hline IFA & PC 38:5.1 & 0.2949042 & 0.1473133 \\
\hline IFA & PC 38:6 & 7.1234569 & 1.5630188 \\
\hline IFA & PC 38:7 & 0.0066066 & 0.0029699 \\
\hline
\end{tabular}




\begin{tabular}{|c|c|c|c|}
\hline IFA & PC 40:4 & 0.0253843 & 0.0090688 \\
\hline IFA & PC 40:5 & 0.2499824 & 0.0757798 \\
\hline IFA & PC 40:5.1 & 0.0333164 & 0.0131165 \\
\hline IFA & PC 40:6.1 & 1.1147742 & 0.3396461 \\
\hline IFA & PC 40:7 & 0.0089394 & 0.0311764 \\
\hline IFA & PC 40:8 & 0.0146751 & 0.0043687 \\
\hline IFA & PC 32:0 o & 0.0275527 & 0.0086761 \\
\hline IFA & PC 34:0 o & 0.0027981 & 0.0009148 \\
\hline IFA & PC 32:1 p & 0.0061988 & 0.0024647 \\
\hline IFA & PC 34:1 p & 0.0788858 & 0.0274006 \\
\hline IFA & PC $34: 2 \mathrm{p}$ & 0.1204576 & 0.0295573 \\
\hline IFA & PC 36:1 p & 0.0041282 & 0.0010508 \\
\hline IFA & PC $36: 2 p$ & 0.0153018 & 0.0035698 \\
\hline IFA & PC 36:4 p & 0.3511014 & 0.106525 \\
\hline IFA & PC 38:3 p & 0.081097 & 0.0204898 \\
\hline IFA & PC 38:4 p & 0.2806301 & 0.0864274 \\
\hline IFA & PC 38:4 p.1 & 0.0503431 & 0.0153801 \\
\hline IFA & PC 38:5 p & 0.1342734 & 0.0422402 \\
\hline IFA & PC $40: 3 p$ & 0.0121976 & 0.0030706 \\
\hline IFA & PC 40:4 p & 0.0214711 & 0.0055188 \\
\hline IFA & PC 40:5 p & 0.0368656 & 0.0113642 \\
\hline IFA & PC 40:6 p & 0.0971024 & 0.0354183 \\
\hline IFA & PC 40:6 p.1 & 0.0334665 & 0.009923 \\
\hline IFA & PC 42:5 p & 0.0065463 & 0.0025268 \\
\hline IFA & PE 34:3 & 0.1146389 & 0.0599862 \\
\hline IFA & PE 38:7 & 0.3375418 & 0.1415107 \\
\hline IFA & PE 34:2 & 0.0919267 & 0.0620603 \\
\hline IFA & PE 36:1 & 0.0300185 & 0.0232051 \\
\hline IFA & PE 36:4 & 0.0843725 & 0.0622276 \\
\hline IFA & PE 38:6 & 0.2139939 & 0.1331927 \\
\hline IFA & PE 34:1 p & 0.0425784 & 0.0184177 \\
\hline IFA & PE 36:2 p & 0.1046856 & 0.0617247 \\
\hline IFA & PE 36:4 p & 0.3991413 & 0.1958227 \\
\hline IFA & PE 38:4 p & 0.3761954 & 0.1745742 \\
\hline IFA & PE 38:5 p & 0.1951929 & 0.0875122 \\
\hline IFA & PE 40:5 p & 0.0485444 & 0.0215723 \\
\hline IFA & SM 37:1 d & 0.007616 & 0.0023158 \\
\hline IFA & SM 38:0 d & 0.0088763 & 0.0060071 \\
\hline IFA & SM 39:2 d & 0.0052143 & 0.0014382 \\
\hline IFA & SM 42:0 d & 0.0047196 & 0.0027203 \\
\hline IFA & SM 44:2 d & 0.004052 & 0.0021327 \\
\hline IFA & SM 42:1 d & 0.0310399 & 0.0213524 \\
\hline IFA & SM 30:1 d & 0.0069589 & 0.0043363 \\
\hline
\end{tabular}




\begin{tabular}{|c|c|c|c|}
\hline IFA & SM 32:0 d & 0.0039972 & 0.0028345 \\
\hline IFA & SM 32:2 d & 0.0097454 & 0.0048926 \\
\hline IFA & SM 34:0 d & 0.0624555 & 0.0215191 \\
\hline IFA & SM 34:2 d & 0.1605549 & 0.0327618 \\
\hline IFA & SM 36:0 d & 0.0315585 & 0.0254066 \\
\hline IFA & SM 36:1 d & 0.2317237 & 0.0722986 \\
\hline IFA & SM 36:2 d & 0.2964634 & 0.0623167 \\
\hline IFA & SM 36:3 d & 0.0037257 & 0.0012834 \\
\hline IFA & SM 38:1 d & 0.1294476 & 0.0353464 \\
\hline IFA & SM 38:2 d & 0.0740422 & 0.0151675 \\
\hline IFA & SM 40:0 d & 0.0128439 & 0.0073279 \\
\hline IFA & SM 40:1 d & 0.2706064 & 0.06438 \\
\hline IFA & SM 40:2 d & 0.139354 & 0.0274346 \\
\hline IFA & SM 42:2 d & 0.6394989 & 0.2165979 \\
\hline IFA & SM 42:3 d & 0.3308828 & 0.0801847 \\
\hline IFA & SM 43:1 d & 0.0017848 & 0.0010368 \\
\hline IFA & TG 52:4 & 0.0332697 & 0.0144661 \\
\hline IFA & TG 40:0 & 0.007016 & 0.0054923 \\
\hline IFA & TG 42:0 & 0.0106121 & 0.0095075 \\
\hline IFA & TG 42:1 & 0.0060958 & 0.0056794 \\
\hline IFA & TG 42:2 & 0.0046848 & 0.0032918 \\
\hline IFA & TG $42: 3$ & 0.0038745 & 0.0035986 \\
\hline IFA & TG 44:0 & 0.0256535 & 0.0191692 \\
\hline IFA & TG 44:1 & 0.0240785 & 0.0266836 \\
\hline IFA & TG 46:0 & 0.1032078 & 0.0600261 \\
\hline IFA & TG 46:1 & 0.0809097 & 0.0676569 \\
\hline IFA & TG 46:2 & 0.0360252 & 0.0316921 \\
\hline IFA & TG 46:3 & 0.0025536 & 0.0016564 \\
\hline IFA & TG 48:1 & 0.5659861 & 0.3226701 \\
\hline IFA & TG 48:2 & 0.1440778 & 0.0914472 \\
\hline IFA & TG 48:3 & 0.0408615 & 0.0288546 \\
\hline IFA & TG 48:4 & 0.0066347 & 0.0055146 \\
\hline IFA & TG 49:1 & 0.0041158 & 0.0020547 \\
\hline IFA & TG 49:2 & 0.0158728 & 0.0073096 \\
\hline IFA & TG 49:3 & 0.0033593 & 0.0016306 \\
\hline IFA & TG 50:0 & 0.0590218 & 0.0302177 \\
\hline IFA & TG 50:1 & 1.0021554 & 1.0765837 \\
\hline IFA & TG 50:2 & 1.2092873 & 0.5671286 \\
\hline IFA & TG 50:3 & 0.2525405 & 0.14027 \\
\hline IFA & TG 50:4 & 0.0419162 & 0.0265651 \\
\hline IFA & TG 50:5 & 0.0039582 & 0.0024576 \\
\hline IFA & TG 51:1 & 0.019165 & 0.0089742 \\
\hline IFA & TG $51: 2$ & 0.0363267 & 0.018948 \\
\hline
\end{tabular}




\begin{tabular}{|c|c|c|c|}
\hline IFA & TG 51:3 & 0.0186234 & 0.0094593 \\
\hline IFA & TG 51:4 & 0.0035598 & 0.0020191 \\
\hline IFA & TG 52:1 & 0.2406567 & 0.1373493 \\
\hline IFA & TG 52:2 & 2.1983564 & 1.5269972 \\
\hline IFA & TG 52:3 & 2.592215 & 1.1982277 \\
\hline IFA & TG 52:4.1 & 0.6601893 & 0.3405633 \\
\hline IFA & TG 52:5 & 0.0345012 & 0.0222165 \\
\hline IFA & TG 52:6 & 0.0066296 & 0.0051064 \\
\hline IFA & TG 53:1 & 0.0070831 & 0.0026819 \\
\hline IFA & TG 53:2 & 0.0217316 & 0.0121459 \\
\hline IFA & TG 53:3 & 0.0211441 & 0.0102973 \\
\hline IFA & TG 53:4 & 0.0105091 & 0.0044903 \\
\hline IFA & TG 54:1 & 0.0278167 & 0.0144222 \\
\hline IFA & TG 54:2 & 0.1824766 & 0.1045102 \\
\hline IFA & TG 54:3 & 0.7642644 & 0.4227972 \\
\hline IFA & TG 54:4 & 0.525667 & 0.3337828 \\
\hline IFA & TG 54:5 & 0.1624144 & 0.0669403 \\
\hline IFA & TG 54:6 & 0.0637448 & 0.0338624 \\
\hline IFA & TG $56: 3$ & 0.0116036 & 0.0062336 \\
\hline IFA & TG $56: 4$ & 0.0168888 & 0.0131314 \\
\hline IFA & TG 56:5 & 0.0390634 & 0.0200898 \\
\hline IFA & TG 56:6 & 0.1072349 & 0.0411735 \\
\hline IFA & TG 56:7 & 0.1827324 & 0.1075734 \\
\hline IFA & TG 56:8 & 0.0016791 & 0.0014794 \\
\hline IFA & TG 57:1 & 0.0083727 & 0.0071137 \\
\hline IFA & TG 58:1 & 0.004774 & 0.003442 \\
\hline IFA & TG $58: 3$ & 0.0030075 & 0.0020274 \\
\hline IFA & TG 58:6 & 0.0055418 & 0.0025839 \\
\hline IFA & TG 58:8 & 0.0047328 & 0.0036193 \\
\hline IFA & TG 58:9 & 0.0309698 & 0.0200027 \\
\hline IFA & Ceramide $32: 1 \mathrm{~d}$ & 0.0002928 & 0.0001133 \\
\hline IFA & Ceramide $34: 0 \mathrm{~d}$ & 0.0006396 & 0.0002646 \\
\hline IFA & Ceramide $34: 1 \mathrm{~d}$ & 0.0027725 & 0.0008183 \\
\hline IFA & Ceramide $34: 2 \mathrm{~d}$ & 0.0002763 & 0.0000688 \\
\hline IFA & Ceramide $36: 1 \mathrm{~d}$ & 0.001005 & 0.0004663 \\
\hline IFA & Ceramide $38: 1 \mathrm{~d}$ & 0.000695 & 0.0002562 \\
\hline IFA & Ceramide 39:1 d & 0.0002916 & 0.0001141 \\
\hline IFA & Ceramide 40:0 d & 0.0012619 & 0.0005959 \\
\hline IFA & Ceramide $40: 1 \mathrm{~d}$ & 0.0033829 & 0.0009764 \\
\hline IFA & Ceramide $40: 2 \mathrm{~d}$ & 0.0004708 & 0.0001347 \\
\hline IFA & Ceramide $41: 1 \mathrm{~d}$ & 0.0024802 & 0.0007011 \\
\hline IFA & Ceramide $42: 0 \mathrm{~d}$ & 0.0019383 & 0.0009137 \\
\hline IFA & Ceramide $42: 1 \mathrm{~d}$ & 0.0159552 & 0.0060927 \\
\hline
\end{tabular}




\begin{tabular}{|c|c|c|c|}
\hline IFA & Ceramide $44: 1 \mathrm{~d}$ & 0.0002671 & 0.0001076 \\
\hline IFA & FA 12:0 & 0.0066281 & 0.0071516 \\
\hline IFA & FA 13:0 & 0.0009401 & 0.0004714 \\
\hline IFA & FA 14:0 & 0.0287537 & 0.0217693 \\
\hline IFA & FA 14:1 & 0.0010194 & 0.0006833 \\
\hline IFA & FA 15:0 & 0.0161904 & 0.0085919 \\
\hline IFA & FA 15:1 & 0.0016708 & 0.0010802 \\
\hline IFA & FA 16:0 & 1.9198203 & 1.4006561 \\
\hline IFA & FA 16:1 & 0.0191451 & 0.0123539 \\
\hline IFA & FA 17:0 & 0.0247965 & 0.014157 \\
\hline IFA & FA 18:1 & 0.1646577 & 0.1001472 \\
\hline IFA & FA 18:2 & 0.0313803 & 0.015915 \\
\hline IFA & FA 18:3 & 0.0009323 & 0.0004946 \\
\hline IFA & FA 20:0 & 0.0533295 & 0.0344251 \\
\hline IFA & FA 20:1 & 0.0057359 & 0.0027089 \\
\hline IFA & FA 20:2 & 0.0011786 & 0.0008093 \\
\hline IFA & FA 20:3 & 0.0003761 & 0.0002074 \\
\hline IFA & FA 20:3.1 & 0.0007403 & 0.000316 \\
\hline IFA & FA 20:4 & 0.0023513 & 0.0008496 \\
\hline IFA & FA 20:5 & 0.0007189 & 0.0004577 \\
\hline IFA & FA 22:1 & 0.0111035 & 0.0065041 \\
\hline IFA & FA 22:2 & 0.0005387 & 0.0002785 \\
\hline IFA & FA 22:6 & 0.0015892 & 0.0007912 \\
\hline IFA & FA 24:0 & 0.0295301 & 0.0118436 \\
\hline IFA & FA 24:1 & 0.0028836 & 0.0017223 \\
\hline IFA & FA 26:0 & 0.0102839 & 0.0050825 \\
\hline IFA & FA 28:0 & 0.0034348 & 0.0017696 \\
\hline IFA & GlcCer 40:1 d.1 & 0.0026785 & 0.0007077 \\
\hline IFA & GlcCer 41:1 d & 0.0014515 & 0.0003318 \\
\hline IFA & GlcCer 42:1 d.1 & 0.0052196 & 0.0014145 \\
\hline IFA & GlcCer $14: 1 \mathrm{~d}$ & 0.0002688 & 0.0000843 \\
\hline IFA & LPC 18:1 & 0.0074064 & 0.0067438 \\
\hline IFA & LPE 20:4 & 0.0017508 & 0.0007614 \\
\hline IFA & PC $34: 1$ ox & 0.1782963 & 0.120497 \\
\hline IFA & PC $36: 1$ ox & 0.0894071 & 0.0639421 \\
\hline IFA & PC $36: 3$ ox & 0.0760728 & 0.0494009 \\
\hline IFA & PC $36: 4$ ox & 0.0108022 & 0.0096028 \\
\hline IFA & PC 38:3 ox & 0.0342822 & 0.0228373 \\
\hline IFA & PC $38: 5$ ox & 0.0875128 & 0.0548806 \\
\hline IFA & PC 32:2 & 0.041125 & 0.0218242 \\
\hline IFA & PC 33:1.1 & 0.0120778 & 0.0041261 \\
\hline IFA & PC 34:3.1 & 0.0423983 & 0.0143412 \\
\hline IFA & PC 34:4 & 0.0123839 & 0.0064009 \\
\hline
\end{tabular}




\begin{tabular}{|c|c|c|c|}
\hline IFA & PC $35: 1.1$ & 0.0183162 & 0.0046394 \\
\hline IFA & PC 35:2.1 & 0.0471128 & 0.0104684 \\
\hline IFA & PC 35:4.1 & 0.0065557 & 0.0013707 \\
\hline IFA & PC 36:3.3 & 0.7124727 & 0.1437203 \\
\hline IFA & PC 36:5.2 & 0.0053601 & 0.002698 \\
\hline IFA & PC 37:2 & 0.0109624 & 0.002906 \\
\hline IFA & PC 38:3.1 & 0.3452416 & 0.0877722 \\
\hline IFA & PC 40:4.1 & 0.0237669 & 0.0086024 \\
\hline IFA & PC 40:5.2 & 0.0207383 & 0.00731 \\
\hline IFA & PC 40:6.2 & 0.0052069 & 0.0020789 \\
\hline IFA & PC 40:8.1 & 0.0144949 & 0.003709 \\
\hline IFA & PC 32:0 p.1 & 0.0224867 & 0.0059452 \\
\hline IFA & PC $34: 0 \mathrm{p}$ & 0.049042 & 0.0107566 \\
\hline IFA & PC 34:1 p.1 & 0.0301399 & 0.0081112 \\
\hline IFA & PC 34:2 p.1 & 0.083143 & 0.0155139 \\
\hline IFA & PC 36:1 p.1 & 0.013587 & 0.0035097 \\
\hline IFA & PC 36:3 p.1 & 0.2078383 & 0.0669219 \\
\hline IFA & PC 36:4 p.1 & 0.1552032 & 0.0535158 \\
\hline IFA & PC 38:4 p.2 & 0.1615937 & 0.0640932 \\
\hline IFA & PC 38:5 p.1 & 0.0370738 & 0.0165683 \\
\hline IFA & PC 40:3 p.1 & 0.0112956 & 0.0026442 \\
\hline IFA & PC 40:4 p.1 & 0.0171801 & 0.0050821 \\
\hline IFA & PC 42:4 p & 0.0142124 & 0.0041205 \\
\hline IFA & PC 42:5 p.1 & 0.0053381 & 0.0018361 \\
\hline IFA & PC 44:4 p & 0.0196414 & 0.0054217 \\
\hline IFA & PE 34:1 & 0.0195649 & 0.0193494 \\
\hline IFA & PE 36:2 & 0.0857042 & 0.0536013 \\
\hline IFA & PE 36:3 & 0.0296203 & 0.0215375 \\
\hline IFA & PE 36:4.1 & 0.0588403 & 0.0583922 \\
\hline IFA & PE 38:2 & 0.1145517 & 0.0500675 \\
\hline IFA & PE 38:4 & 0.109448 & 0.0790307 \\
\hline IFA & PE 38:6.1 & 0.1090591 & 0.0714314 \\
\hline IFA & PE 40:6 & 0.0578348 & 0.0233688 \\
\hline IFA & PE 34:1 p.1 & 0.0336497 & 0.015536 \\
\hline IFA & PE 34:2 p & 0.0649856 & 0.0326308 \\
\hline IFA & PE 36:1 p & 0.0245823 & 0.0138358 \\
\hline IFA & PE 36:2 p.1 & 0.088318 & 0.0536387 \\
\hline IFA & PE 36:4 p.1 & 0.320295 & 0.1646583 \\
\hline IFA & PE 36:5 p & 0.0511178 & 0.03858 \\
\hline IFA & PE 38:3 p & 0.0174866 & 0.0110981 \\
\hline IFA & PE 38:4 p.1 & 0.3041991 & 0.1729397 \\
\hline IFA & PE 38:5 p.1 & 0.1340207 & 0.0639391 \\
\hline IFA & PE 38:6 p & 0.201743 & 0.0900529 \\
\hline
\end{tabular}




\begin{tabular}{|c|c|c|c|}
\hline IFA & PE 40:4 p & 0.0417752 & 0.0204789 \\
\hline IFA & PE 40:5 p.1 & 0.0236518 & 0.0112022 \\
\hline IFA & PE 40:6 p & 0.1680759 & 0.06853 \\
\hline IFA & PE 40:7 p & 0.0818823 & 0.0371542 \\
\hline IFA & PE 40:6.1 & 0.070704 & 0.0521237 \\
\hline IFA & SM 32:1 d & 0.6040555 & 0.2432702 \\
\hline IFA & SM 33:1 d & 0.1027727 & 0.0272922 \\
\hline IFA & SM 34:1 d & 0.1409922 & 0.0206633 \\
\hline IFA & SM 39:1 d & 0.1490923 & 0.0357333 \\
\hline IFA & SM 40:2 d.1 & 0.597116 & 0.1536945 \\
\hline IFA & SM 40:3 d & 0.039853 & 0.0158486 \\
\hline IFA & SM $41: 1 \mathrm{~d}$ & 0.5657862 & 0.1712711 \\
\hline IFA & SM 41:2 d & 0.2437669 & 0.0814548 \\
\hline IFA & SM 42:1 d.1 & 1.8715929 & 0.5188128 \\
\hline IFA & SM 43:2 d & 0.0258866 & 0.0088591 \\
\hline SQ-LNS & CE 16:1 & 0.1172686 & 0.0375424 \\
\hline SQ-LNS & CE 18:1 & 2.5704347 & 0.8078552 \\
\hline SQ-LNS & CE 18:2 & 16.5461683 & 3.7542437 \\
\hline SQ-LNS & CE 18:3 & 0.1422087 & 0.0491212 \\
\hline SQ-LNS & CE 20:3 & 0.2404385 & 0.0767924 \\
\hline SQ-LNS & CE 20:4 & 1.9353538 & 0.8541507 \\
\hline SQ-LNS & CE 20:5 & 0.4098558 & 0.2457458 \\
\hline SQ-LNS & CE 22:6 & 0.6411996 & 0.2085378 \\
\hline SQ-LNS & Ceramide $42: 2 \mathrm{~d}$ & 0.0084946 & 0.0293267 \\
\hline SQ-LNS & Cholesterol & 7.9439618 & 1.0939401 \\
\hline SQ-LNS & DG 32:1 & 0.0306967 & 0.0196531 \\
\hline SQ-LNS & DG 34:1 & 0.1886315 & 0.2178358 \\
\hline SQ-LNS & DG 34:2 & 0.262915 & 0.8738713 \\
\hline SQ-LNS & DG 34:3 & 0.0078732 & 0.0051651 \\
\hline SQ-LNS & DG 36:1 & 0.0355209 & 0.0699222 \\
\hline SQ-LNS & DG 36:2 & 0.240809 & 0.5201445 \\
\hline SQ-LNS & DG 36:3 & 0.2203406 & 0.1780222 \\
\hline SQ-LNS & DG 36:4 A & 0.0445387 & 0.045145 \\
\hline SQ-LNS & DG 38:5 & 0.0552547 & 0.1400318 \\
\hline SQ-LNS & DG 38:6 & 0.0297175 & 0.0429936 \\
\hline SQ-LNS & GlcCer 40:1 d & 0.006362 & 0.0015482 \\
\hline SQ-LNS & GlcCer 42:1 d & 0.010395 & 0.002391 \\
\hline SQ-LNS & GlcCer 42:2 d & 0.0107109 & 0.0028466 \\
\hline SQ-LNS & Gal-Gal-Cer 42:2 d & 0.0009497 & 0.0004893 \\
\hline SQ-LNS & LPC 20:0 & 0.0013124 & 0.0006989 \\
\hline SQ-LNS & LPC 20:1 & 0.0010376 & 0.0006871 \\
\hline SQ-LNS & LPC 22:4 & 0.0013313 & 0.0013149 \\
\hline SQ-LNS & LPC 22:6 & 0.0030319 & 0.0034239 \\
\hline
\end{tabular}




\begin{tabular}{|c|c|c|c|}
\hline SQ-LNS & LPC 16:0 o & 0.0037945 & 0.0034384 \\
\hline SQ-LNS & LPC $16: 0 \mathrm{p}$ & 0.0027348 & 0.0018153 \\
\hline SQ-LNS & LPC 16:0 & 0.0477863 & 0.0278614 \\
\hline SQ-LNS & LPC 18:0 & 0.0866232 & 0.0450481 \\
\hline SQ-LNS & LPC 18:2 & 0.0072848 & 0.0138528 \\
\hline SQ-LNS & LPC 20:3 & 0.0009618 & 0.0013843 \\
\hline SQ-LNS & LPC 20:4 & 0.0043289 & 0.0044051 \\
\hline SQ-LNS & LPC 20:5 & 0.0005992 & 0.0005718 \\
\hline SQ-LNS & PC 25:0 & 0.0048714 & 0.0038456 \\
\hline SQ-LNS & PC $37: 3$ & 0.0068339 & 0.0022003 \\
\hline SQ-LNS & PC 39:6 & 0.0192601 & 0.0095333 \\
\hline SQ-LNS & PC 42:5 & 0.0038281 & 0.0017599 \\
\hline SQ-LNS & PC 42:6 & 0.0018966 & 0.0011516 \\
\hline SQ-LNS & PC $32: 0 \mathrm{p}$ & 0.0336667 & 0.0101927 \\
\hline SQ-LNS & PC $36: 3 p$ & 0.265237 & 0.0733309 \\
\hline SQ-LNS & PC $38: 2 \mathrm{p}$ & 0.0071739 & 0.0020064 \\
\hline SQ-LNS & PC $42: 3 p$ & 0.0069965 & 0.0023865 \\
\hline SQ-LNS & PC 34:1 & 0.0623357 & 0.0193641 \\
\hline SQ-LNS & PC 36:3 & 0.0743385 & 0.0155648 \\
\hline SQ-LNS & PC 36:5 & 0.6194141 & 0.143297 \\
\hline SQ-LNS & PC 38:4 & 0.0351583 & 0.0103654 \\
\hline SQ-LNS & PC 40:6 & 0.0265605 & 0.0096341 \\
\hline SQ-LNS & PC 28:0 & 0.005046 & 0.0034233 \\
\hline SQ-LNS & PC 30:0 & 0.1312689 & 0.0783405 \\
\hline SQ-LNS & PC 31:0 & 0.0033269 & 0.001386 \\
\hline SQ-LNS & PC 32:0 & 0.1653747 & 0.0671799 \\
\hline SQ-LNS & PC 32:1 & 0.1588391 & 0.0835923 \\
\hline SQ-LNS & PC 33:0 & 0.0044926 & 0.002161 \\
\hline SQ-LNS & PC 33:1 & 0.0230748 & 0.0095425 \\
\hline SQ-LNS & PC 33:2 & 0.023875 & 0.0082652 \\
\hline SQ-LNS & PC 34:0 & 0.0308811 & 0.0055635 \\
\hline SQ-LNS & PC 34:1.1 & 5.4348017 & 1.9297814 \\
\hline SQ-LNS & PC 34:2 & 12.4044609 & 2.7498819 \\
\hline SQ-LNS & PC $34: 3$ & 0.0237397 & 0.018558 \\
\hline SQ-LNS & PC 35:1 & 0.0251091 & 0.0081639 \\
\hline SQ-LNS & PC 35:2 & 0.0614211 & 0.0262102 \\
\hline SQ-LNS & PC 35:3 & 0.0132715 & 0.0052922 \\
\hline SQ-LNS & PC 35:4 & 0.0069946 & 0.0019665 \\
\hline SQ-LNS & PC 36:1 & 0.5897235 & 0.2094777 \\
\hline SQ-LNS & PC 36:2 & 6.0514834 & 1.5973228 \\
\hline SQ-LNS & PC 36:3.1 & 0.9733252 & 0.3439876 \\
\hline SQ-LNS & PC $36: 3.2$ & 1.0874944 & 0.3132306 \\
\hline SQ-LNS & PC 36:4 & 0.2041814 & 0.0973363 \\
\hline
\end{tabular}




\begin{tabular}{|c|c|c|c|}
\hline SQ-LNS & PC 36:4.1 & 0.0721607 & 0.0339318 \\
\hline SQ-LNS & PC 36:5.1 & 0.0393919 & 0.1296014 \\
\hline SQ-LNS & PC 36:6 & 0.0121756 & 0.006808 \\
\hline SQ-LNS & PC 37:4 & 0.0186188 & 0.0103226 \\
\hline SQ-LNS & PC 37:6 & 0.0063777 & 0.0019863 \\
\hline SQ-LNS & PC 38:2 & 0.0397587 & 0.0093036 \\
\hline SQ-LNS & PC $38: 3$ & 0.4119036 & 0.1217888 \\
\hline SQ-LNS & PC 38:4.1 & 0.0810662 & 0.0285069 \\
\hline SQ-LNS & PC 38:5 & 0.6215453 & 0.189588 \\
\hline SQ-LNS & PC 38:5.1 & 0.2952537 & 0.1508099 \\
\hline SQ-LNS & PC 38:6 & 7.1127158 & 1.9166711 \\
\hline SQ-LNS & PC 38:7 & 0.0066044 & 0.0034111 \\
\hline SQ-LNS & PC 40:4 & 0.0248568 & 0.0077324 \\
\hline SQ-LNS & PC 40:5 & 0.2616236 & 0.0900439 \\
\hline SQ-LNS & PC 40:5.1 & 0.0323922 & 0.0134681 \\
\hline SQ-LNS & PC 40:6.1 & 1.2156765 & 0.4251956 \\
\hline SQ-LNS & PC 40:7 & 0.0126845 & 0.0275439 \\
\hline SQ-LNS & PC 40:8 & 0.0134552 & 0.0050849 \\
\hline SQ-LNS & PC 32:0 o & 0.0281432 & 0.0101377 \\
\hline SQ-LNS & PC 34:0 o & 0.0028988 & 0.00106 \\
\hline SQ-LNS & PC 32:1 p & 0.006231 & 0.0019771 \\
\hline SQ-LNS & PC $34: 1 \mathrm{p}$ & 0.0757199 & 0.0247489 \\
\hline SQ-LNS & PC $34: 2 \mathrm{p}$ & 0.1119818 & 0.0288562 \\
\hline SQ-LNS & PC $36: 1 \mathrm{p}$ & 0.0040475 & 0.0011834 \\
\hline SQ-LNS & PC $36: 2 \mathrm{p}$ & 0.0140285 & 0.0032869 \\
\hline SQ-LNS & PC $36: 4 \mathrm{p}$ & 0.3457668 & 0.1184345 \\
\hline SQ-LNS & PC $38: 3$ p & 0.079174 & 0.0206566 \\
\hline SQ-LNS & PC 38:4 p & 0.2729425 & 0.0811178 \\
\hline SQ-LNS & PC 38:4 p.1 & 0.0490453 & 0.0146622 \\
\hline SQ-LNS & PC 38:5 p & 0.1369679 & 0.0435463 \\
\hline SQ-LNS & PC $40: 3 p$ & 0.0113999 & 0.0034688 \\
\hline SQ-LNS & PC 40:4 p & 0.0208587 & 0.0056971 \\
\hline SQ-LNS & PC $40: 5 p$ & 0.0376691 & 0.0103214 \\
\hline SQ-LNS & PC 40:6 p & 0.0960779 & 0.0340754 \\
\hline SQ-LNS & PC 40:6 p.1 & 0.0360321 & 0.0130526 \\
\hline SQ-LNS & PC $42: 5 p$ & 0.0056704 & 0.0025689 \\
\hline SQ-LNS & PE 34:3 & 0.1148354 & 0.0742051 \\
\hline SQ-LNS & PE 38:7 & 0.4085992 & 0.2686413 \\
\hline SQ-LNS & PE 34:2 & 0.0834202 & 0.0403411 \\
\hline SQ-LNS & PE 36:1 & 0.0279161 & 0.0130278 \\
\hline SQ-LNS & PE 36:4 & 0.070651 & 0.0298363 \\
\hline SQ-LNS & PE 38:6 & 0.1940924 & 0.0876449 \\
\hline SQ-LNS & PE 34:1 p & 0.0446616 & 0.0327414 \\
\hline
\end{tabular}




\begin{tabular}{|c|c|c|c|}
\hline SQ-LNS & PE 36:2 p & 0.1029268 & 0.0592955 \\
\hline SQ-LNS & PE 36:4 p & 0.4953766 & 0.3926091 \\
\hline SQ-LNS & PE 38:4 p & 0.4232692 & 0.2942923 \\
\hline SQ-LNS & PE 38:5 p & 0.2318585 & 0.1721598 \\
\hline SQ-LNS & PE 40:5 p & 0.0535427 & 0.030376 \\
\hline SQ-LNS & SM 37:1 d & 0.0082721 & 0.0021362 \\
\hline SQ-LNS & SM 38:0 d & 0.0084384 & 0.0034748 \\
\hline SQ-LNS & SM 39:2 d & 0.0058114 & 0.0014984 \\
\hline SQ-LNS & SM 42:0 d & 0.0040643 & 0.0016704 \\
\hline SQ-LNS & SM 44:2 d & 0.0040925 & 0.001768 \\
\hline SQ-LNS & SM 42:1 d & 0.0274527 & 0.0114634 \\
\hline SQ-LNS & SM 30:1 d & 0.0067356 & 0.0034033 \\
\hline SQ-LNS & SM 32:0 d & 0.0035501 & 0.0018594 \\
\hline SQ-LNS & SM 32:2 d & 0.0096374 & 0.0038489 \\
\hline SQ-LNS & SM 34:0 d & 0.0583558 & 0.0178946 \\
\hline SQ-LNS & SM 34:2 d & 0.1582544 & 0.0336282 \\
\hline SQ-LNS & SM 36:0 d & 0.0272507 & 0.012595 \\
\hline SQ-LNS & SM 36:1 d & 0.2285555 & 0.0625031 \\
\hline SQ-LNS & SM 36:2 d & 0.2886074 & 0.075667 \\
\hline SQ-LNS & SM 36:3 d & 0.0036113 & 0.0013661 \\
\hline SQ-LNS & SM 38:1 d & 0.1346627 & 0.0294519 \\
\hline SQ-LNS & SM 38:2 d & 0.0719533 & 0.0161779 \\
\hline SQ-LNS & SM 40:0 d & 0.0120294 & 0.0052342 \\
\hline SQ-LNS & SM 40:1 d & 0.2754813 & 0.0549353 \\
\hline SQ-LNS & SM 40:2 d & 0.1443573 & 0.0278784 \\
\hline SQ-LNS & SM 42:2 d & 0.6287308 & 0.1752583 \\
\hline SQ-LNS & SM 42:3 d & 0.3302789 & 0.071043 \\
\hline SQ-LNS & SM 43:1 d & 0.001799 & 0.0011482 \\
\hline SQ-LNS & TG 52:4 & 0.0332221 & 0.0129615 \\
\hline SQ-LNS & TG 40:0 & 0.0078556 & 0.0046118 \\
\hline SQ-LNS & TG 42:0 & 0.0107287 & 0.0070818 \\
\hline SQ-LNS & TG 42:1 & 0.0068933 & 0.0054528 \\
\hline SQ-LNS & TG 42:2 & 0.0050585 & 0.0035522 \\
\hline SQ-LNS & TG $42: 3$ & 0.0047871 & 0.0050918 \\
\hline SQ-LNS & TG 44:0 & 0.0261402 & 0.0150106 \\
\hline SQ-LNS & TG 44:1 & 0.0264604 & 0.0221281 \\
\hline SQ-LNS & TG 46:0 & 0.105516 & 0.0543367 \\
\hline SQ-LNS & TG 46:1 & 0.0867889 & 0.0616148 \\
\hline SQ-LNS & TG 46:2 & 0.0379196 & 0.0273824 \\
\hline SQ-LNS & TG 46:3 & 0.003257 & 0.0021997 \\
\hline SQ-LNS & TG 48:1 & 0.5626962 & 0.2925713 \\
\hline SQ-LNS & TG 48:2 & 0.1438373 & 0.0804847 \\
\hline SQ-LNS & TG 48:3 & 0.0460452 & 0.0327937 \\
\hline
\end{tabular}




\begin{tabular}{|c|c|c|c|}
\hline SQ-LNS & TG 48:4 & 0.0083208 & 0.0071226 \\
\hline SQ-LNS & TG 49:1 & 0.0041954 & 0.0018325 \\
\hline SQ-LNS & TG 49:2 & 0.0169838 & 0.0075757 \\
\hline SQ-LNS & TG 49:3 & 0.0033585 & 0.0023448 \\
\hline SQ-LNS & TG 50:0 & 0.058592 & 0.0267954 \\
\hline SQ-LNS & TG 50:1 & 0.9162823 & 0.8320556 \\
\hline SQ-LNS & TG 50:2 & 1.1478158 & 0.4073598 \\
\hline SQ-LNS & TG 50:3 & 0.2417879 & 0.1049906 \\
\hline SQ-LNS & TG 50:4 & 0.042297 & 0.0238009 \\
\hline SQ-LNS & TG 50:5 & 0.0044362 & 0.0023304 \\
\hline SQ-LNS & TG 51:1 & 0.019202 & 0.0079921 \\
\hline SQ-LNS & TG 51:2 & 0.0341554 & 0.0138308 \\
\hline SQ-LNS & TG 51:3 & 0.0174202 & 0.0062931 \\
\hline SQ-LNS & TG 51:4 & 0.0037143 & 0.0015339 \\
\hline SQ-LNS & TG 52:1 & 0.2348302 & 0.1103584 \\
\hline SQ-LNS & TG 52:2 & 2.248459 & 1.4384472 \\
\hline SQ-LNS & TG 52:3 & 2.5911007 & 0.9890893 \\
\hline SQ-LNS & TG 52:4.1 & 0.6914702 & 0.3350214 \\
\hline SQ-LNS & TG 52:5 & 0.0359948 & 0.0194739 \\
\hline SQ-LNS & TG 52:6 & 0.006045 & 0.0033895 \\
\hline SQ-LNS & TG 53:1 & 0.007795 & 0.0034793 \\
\hline SQ-LNS & TG 53:2 & 0.0218266 & 0.0112691 \\
\hline SQ-LNS & TG 53:3 & 0.0212971 & 0.0085751 \\
\hline SQ-LNS & TG 53:4 & 0.010304 & 0.0034295 \\
\hline SQ-LNS & TG 54:1 & 0.0315867 & 0.0173837 \\
\hline SQ-LNS & TG 54:2 & 0.1784553 & 0.0890126 \\
\hline SQ-LNS & TG 54:3 & 0.7259255 & 0.3178531 \\
\hline SQ-LNS & TG 54:4 & 0.5246409 & 0.26219 \\
\hline SQ-LNS & TG 54:5 & 0.161007 & 0.0588594 \\
\hline SQ-LNS & TG 54:6 & 0.0611312 & 0.0240471 \\
\hline SQ-LNS & TG 56:3 & 0.0110907 & 0.0048798 \\
\hline SQ-LNS & TG 56:4 & 0.0138708 & 0.0091145 \\
\hline SQ-LNS & TG 56:5 & 0.0413495 & 0.0199761 \\
\hline SQ-LNS & TG 56:6 & 0.1104069 & 0.0390809 \\
\hline SQ-LNS & TG 56:7 & 0.193695 & 0.1161735 \\
\hline SQ-LNS & TG 56:8 & 0.0017132 & 0.0010113 \\
\hline SQ-LNS & TG 57:1 & 0.0065229 & 0.0060171 \\
\hline SQ-LNS & TG 58:1 & 0.0045234 & 0.003258 \\
\hline SQ-LNS & TG 58:3 & 0.0031279 & 0.0017908 \\
\hline SQ-LNS & TG 58:6 & 0.0055387 & 0.0023034 \\
\hline SQ-LNS & TG 58:8 & 0.0045036 & 0.0024864 \\
\hline SQ-LNS & TG 58:9 & 0.0317853 & 0.0172436 \\
\hline SQ-LNS & Ceramide $32: 1 \mathrm{~d}$ & 0.0013969 & 0.0068059 \\
\hline
\end{tabular}




\begin{tabular}{|c|c|c|c|}
\hline SQ-LNS & Ceramide $34: 0 \mathrm{~d}$ & 0.0008794 & 0.0019474 \\
\hline SQ-LNS & Ceramide $34: 1 \mathrm{~d}$ & 0.0121097 & 0.0578162 \\
\hline SQ-LNS & Ceramide $34: 2 \mathrm{~d}$ & 0.0047711 & 0.0283305 \\
\hline SQ-LNS & Ceramide $36: 1 \mathrm{~d}$ & 0.0028992 & 0.0124878 \\
\hline SQ-LNS & Ceramide 38:1 d & 0.0025634 & 0.0117642 \\
\hline SQ-LNS & Ceramide 39:1 d & 0.0007598 & 0.0028978 \\
\hline SQ-LNS & Ceramide 40:0 d & 0.0012374 & 0.0009647 \\
\hline SQ-LNS & Ceramide $40: 1 \mathrm{~d}$ & 0.0068511 & 0.0217862 \\
\hline SQ-LNS & Ceramide $40: 2 \mathrm{~d}$ & 0.0034911 & 0.0189749 \\
\hline SQ-LNS & Ceramide 41:1 d & 0.0034823 & 0.0065247 \\
\hline SQ-LNS & Ceramide 42:0 d & 0.0017815 & 0.0009398 \\
\hline SQ-LNS & Ceramide $42: 1 \mathrm{~d}$ & 0.0187138 & 0.0217754 \\
\hline SQ-LNS & Ceramide $44: 1 \mathrm{~d}$ & 0.000287 & 0.0001911 \\
\hline SQ-LNS & FA 12:0 & 0.0052904 & 0.0049265 \\
\hline SQ-LNS & FA 13:0 & 0.0012885 & 0.0021053 \\
\hline SQ-LNS & FA 14:0 & 0.0341244 & 0.0584196 \\
\hline SQ-LNS & FA $14: 1$ & 0.004127 & 0.0175151 \\
\hline SQ-LNS & FA 15:0 & 0.0337536 & 0.0961399 \\
\hline SQ-LNS & FA 15:1 & 0.0064931 & 0.0262332 \\
\hline SQ-LNS & FA 16:0 & 1.6806879 & 1.4411496 \\
\hline SQ-LNS & FA 16:1 & 0.0668977 & 0.2472031 \\
\hline SQ-LNS & FA 17:0 & 0.0328986 & 0.054919 \\
\hline SQ-LNS & FA 18:1 & 0.1799265 & 0.1857238 \\
\hline SQ-LNS & FA 18:2 & 0.0358925 & 0.0417978 \\
\hline SQ-LNS & FA 18:3 & 0.0015126 & 0.0023479 \\
\hline SQ-LNS & FA 20:0 & 0.0519653 & 0.0491859 \\
\hline SQ-LNS & FA 20:1 & 0.0076104 & 0.0108292 \\
\hline SQ-LNS & FA 20:2 & 0.0033988 & 0.0105294 \\
\hline SQ-LNS & FA 20:3 & 0.0008934 & 0.002721 \\
\hline SQ-LNS & FA 20:3.1 & 0.0008502 & 0.0007836 \\
\hline SQ-LNS & FA 20:4 & 0.0026703 & 0.0021525 \\
\hline SQ-LNS & FA $20: 5$ & 0.0007859 & 0.0006339 \\
\hline SQ-LNS & FA 22:1 & 0.0102528 & 0.006256 \\
\hline SQ-LNS & FA $22: 2$ & 0.0010511 & 0.0025574 \\
\hline SQ-LNS & FA 22:6 & 0.0018178 & 0.00168 \\
\hline SQ-LNS & FA 24:0 & 0.0445648 & 0.0795674 \\
\hline SQ-LNS & FA 24:1 & 0.0031482 & 0.0030622 \\
\hline SQ-LNS & FA 26:0 & 0.0132946 & 0.0170698 \\
\hline SQ-LNS & FA 28:0 & 0.0038908 & 0.0036526 \\
\hline SQ-LNS & GlcCer 40:1 d.1 & 0.0029535 & 0.000727 \\
\hline SQ-LNS & GlcCer 41:1 d & 0.0015315 & 0.0003807 \\
\hline SQ-LNS & GlcCer 42:1 d.1 & 0.0056147 & 0.001429 \\
\hline SQ-LNS & GlcCer $14: 1 \mathrm{~d}$ & 0.0002745 & 0.0000974 \\
\hline
\end{tabular}




\begin{tabular}{|c|c|c|c|}
\hline SQ-LNS & LPC 18:1 & 0.0064346 & 0.0047371 \\
\hline SQ-LNS & LPE 20:4 & 0.0015897 & 0.0007904 \\
\hline SQ-LNS & PC $34: 1$ ox & 0.1633026 & 0.1117518 \\
\hline SQ-LNS & PC 36:1 ox & 0.0869455 & 0.0667646 \\
\hline SQ-LNS & PC $36: 3$ ox & 0.0680817 & 0.0460763 \\
\hline SQ-LNS & PC 36:4 ox & 0.0089308 & 0.0058718 \\
\hline SQ-LNS & PC $38: 3$ ox & 0.0333052 & 0.0245745 \\
\hline SQ-LNS & PC 38:5 ox & 0.0788452 & 0.0466738 \\
\hline SQ-LNS & PC 32:2 & 0.035841 & 0.0166356 \\
\hline SQ-LNS & PC 33:1.1 & 0.0103018 & 0.0042478 \\
\hline SQ-LNS & PC $34: 3.1$ & 0.0384133 & 0.014787 \\
\hline SQ-LNS & PC 34:4 & 0.010816 & 0.0055391 \\
\hline SQ-LNS & PC 35:1.1 & 0.0166963 & 0.0053629 \\
\hline SQ-LNS & PC 35:2.1 & 0.0438978 & 0.0108403 \\
\hline SQ-LNS & PC 35:4.1 & 0.0057735 & 0.0015945 \\
\hline SQ-LNS & PC $36: 3.3$ & 0.6666642 & 0.1950993 \\
\hline SQ-LNS & PC $36: 5.2$ & 0.0047325 & 0.0022712 \\
\hline SQ-LNS & PC $37: 2$ & 0.0107976 & 0.0030357 \\
\hline SQ-LNS & PC 38:3.1 & 0.3513382 & 0.1000436 \\
\hline SQ-LNS & PC 40:4.1 & 0.0227492 & 0.0074492 \\
\hline SQ-LNS & PC 40:5.2 & 0.0191121 & 0.0081442 \\
\hline SQ-LNS & PC 40:6.2 & 0.0046972 & 0.0017273 \\
\hline SQ-LNS & PC 40:8.1 & 0.0132413 & 0.004149 \\
\hline SQ-LNS & PC 32:0 p.1 & 0.0224336 & 0.0059068 \\
\hline SQ-LNS & PC $34: 0 \mathrm{p}$ & 0.0486178 & 0.0154074 \\
\hline SQ-LNS & PC 34:1 p.1 & 0.0268755 & 0.0089101 \\
\hline SQ-LNS & PC $34: 2$ p.1 & 0.0758884 & 0.0183447 \\
\hline SQ-LNS & PC 36:1 p.1 & 0.012438 & 0.0038519 \\
\hline SQ-LNS & PC $36: 3$ p.1 & 0.1951722 & 0.0527709 \\
\hline SQ-LNS & PC 36:4 p.1 & 0.1466776 & 0.0458757 \\
\hline SQ-LNS & PC $38: 4$ p.2 & 0.1466107 & 0.0413566 \\
\hline SQ-LNS & PC 38:5 p.1 & 0.0350587 & 0.0101234 \\
\hline SQ-LNS & PC 40:3 p.1 & 0.0106764 & 0.0032772 \\
\hline SQ-LNS & PC 40:4 p.1 & 0.0161831 & 0.0043761 \\
\hline SQ-LNS & PC $42: 4 \mathrm{p}$ & 0.012957 & 0.0043903 \\
\hline SQ-LNS & PC 42:5 p.1 & 0.004857 & 0.0017338 \\
\hline SQ-LNS & PC $44: 4 \mathrm{p}$ & 0.0184854 & 0.0062327 \\
\hline SQ-LNS & PE 34:1 & 0.0147663 & 0.0076946 \\
\hline SQ-LNS & PE 36:2 & 0.082601 & 0.041408 \\
\hline SQ-LNS & PE 36:3 & 0.0280081 & 0.0161827 \\
\hline SQ-LNS & PE 36:4.1 & 0.046685 & 0.0303738 \\
\hline SQ-LNS & PE 38:2 & 0.1239872 & 0.0771388 \\
\hline SQ-LNS & PE 38:4 & 0.1037742 & 0.0610657 \\
\hline
\end{tabular}




\begin{tabular}{|l|l|r|r|} 
SQ-LNS & PE 38:6.1 & 0.0942571 & 0.044743 \\
\hline SQ-LNS & PE 40:6 & 0.0628568 & 0.0409313 \\
\hline SQ-LNS & PE 34:1 p.1 & 0.0455344 & 0.06819 \\
\hline SQ-LNS & PE 34:2 p & 0.0655866 & 0.0452342 \\
\hline SQ-LNS & PE 36:1 p & 0.0285322 & 0.0307308 \\
\hline SQ-LNS & PE 36:2 p.1 & 0.0837786 & 0.0496689 \\
\hline SQ-LNS & PE 36:4 p.1 & 0.4020645 & 0.3676741 \\
\hline SQ-LNS & PE 36:5 p & 0.0649994 & 0.0530424 \\
\hline SQ-LNS & PE 38:3 p & 0.0182673 & 0.0145774 \\
\hline SQ-LNS & PE 38:4 p.1 & 0.35287 & 0.2757311 \\
\hline SQ-LNS & PE 38:5 p.1 & 0.1680656 & 0.136812 \\
\hline SQ-LNS & PE 38:6 p & 0.2507388 & 0.1802873 \\
\hline SQ-LNS & PE 40:4 p & 0.0432454 & 0.0275944 \\
\hline SQ-LNS & PE 40:5 p.1 & 0.0256717 & 0.0165422 \\
\hline SQ-LNS & PE 40:6 p & 0.2018203 & 0.1421401 \\
\hline SQ-LNS & PE 40:7 p & 0.103705 & 0.0775974 \\
\hline SQ-LNS & PE 40:6.1 & 0.0614635 & 0.0369444 \\
\hline SQ-LNS & SM 32:1 d & 0.6388281 & 0.3067823 \\
\hline SQ-LNS & SM 33:1 d & 0.1008706 & 0.0242546 \\
\hline SQ-LNS & SM 34:1 d & 0.139326 & 0.0294711 \\
\hline SQ-LNS & SM 39:1 d & 0.1617093 & 0.0452437 \\
\hline SQ-LNS & SM 40:2 d.1 & 0.6006079 & 0.2135754 \\
\hline SQ-LNS & SM 40:3 d & 0.0402978 & 0.012407 \\
\hline SQ-LNS & SM 41:1 d & 0.5686648 & 0.15228 \\
\hline SQ-LNS & SM 41:2 d & 0.2463125 & 0.0669829 \\
\hline SQ-LNS & SM 42:1 d.1 & 1.8647426 & 0.4814959 \\
\hline SQ-LNS & SM 43:2 d & 0.0267404 & 0.011101 \\
\hline
\end{tabular}




\begin{tabular}{|l|l|r|r|}
\hline \multicolumn{5}{|l}{ Table S2. 33 HDL Associated Proteins. } \\
\hline Treatment & Protein & Mean Abundance & SD \\
\hline IFA & APOC1 & 10811.85928 & 4721.299434 \\
\hline IFA & APOA1 & 1434574.094 & 537468.0296 \\
\hline IFA & FETUA & 33573.28762 & 17359.22421 \\
\hline IFA & PLTP & 5623.374983 & 4457.277207 \\
\hline IFA & APOD & 329722.0244 & 124745.4338 \\
\hline IFA & SAA4 & 32818.62972 & 11662.22981 \\
\hline IFA & APOL1 & 8561.503842 & 8570.663364 \\
\hline IFA & AACT & 5297.747485 & 3508.021458 \\
\hline IFA & APOE & 61137.52336 & 42382.86114 \\
\hline IFA & A1AT & 61361.18929 & 22378.9738 \\
\hline IFA & APOA2 & 194283.795 & 89854.10462 \\
\hline IFA & C1S & 2074.737315 & 1108.66985 \\
\hline IFA & APOC2 & 242650.1624 & 190106.9337 \\
\hline IFA & APOA4 & 11295.49729 & 9835.211986 \\
\hline IFA & LCAT & 2754.608693 & 1701.719108 \\
\hline IFA & A1BG & 76818.44814 & 51910.24348 \\
\hline IFA & APOC4 & 975.8648138 & 949.1820964 \\
\hline IFA & APOM & 853981.3669 & 288462.2957 \\
\hline IFA & C3 & 398.4395162 & 219.1307649 \\
\hline IFA & SAA2 & 14229.66271 & 9066.199282 \\
\hline IFA & SAA1 & 16262.24619 & 30428.87241 \\
\hline IFA & APOA & 7.557435965 & 6.499382815 \\
\hline IFA & HPX & 7562.57927 & 5447.714586 \\
\hline IFA & APOC3 & 46241.45447 & 57844.54375 \\
\hline IFA & APOF & 1561.646202 & 1008.007114 \\
\hline IFA & HCF2 & 2914.654609 & 1608.458057 \\
\hline IFA & KNG1 & 7765.542371 & 6369.46123 \\
\hline IFA & APOH & 1854.631842 & 1464.540365 \\
\hline IFA & PON1 & 16.96993885 & 21.56828186 \\
\hline IFA & CLUS & 2545.537839 & 1228.904949 \\
\hline IFA & APOB & 4325.351691 & 2240.120226 \\
\hline IFA & PON3 & 643.8255329 & 644.2029852 \\
\hline IFA & APOA5 & 9.084599445 & 6.937561639 \\
\hline SQ-LNS & APOC1 & 24343.81273 & 74660.13338 \\
\hline SQ-LNS & APOA1 & 1527916.768 & 468350.9347 \\
\hline SQ-LNS & FETUA & 30807.90691 & 30708.42266 \\
\hline SQ-LNS & PLTP & 5928.588681 & 3818.181819 \\
\hline SQ-LNS & APOD & 347311.1365 & 137578.4109 \\
\hline SQ-LNS & SAA4 & 36651.03133 & 20392.23984 \\
\hline SQ-LNS & APOL1 & 7836.096267 & 6491.720431 \\
\hline & & & \\
\hline
\end{tabular}




\begin{tabular}{|l|l|r|r|} 
SQ-LNS & AACT & 4511.620293 & 4065.214564 \\
\hline SQ-LNS & APOE & 73933.4948 & 62933.875 \\
\hline SQ-LNS & A1AT & 63538.09406 & 38373.98613 \\
\hline SQ-LNS & APOA2 & 210157.6142 & 105517.1161 \\
\hline SQ-LNS & C1S & 1755.865029 & 1243.31605 \\
\hline SQ-LNS & APOC2 & 252766.0797 & 165642.9704 \\
\hline SQ-LNS & APOA4 & 8757.442428 & 6441.141791 \\
\hline SQ-LNS & LCAT & 3013.289631 & 1983.950556 \\
\hline SQ-LNS & A1BG & 66299.90019 & 75130.04769 \\
\hline SQ-LNS & APOC4 & 1290.926044 & 1192.282178 \\
\hline SQ-LNS & APOM & 804693.8012 & 281472.0627 \\
\hline SQ-LNS & C3 & 471.9884806 & 410.1395085 \\
\hline SQ-LNS & SAA2 & 16249.56888 & 9897.608383 \\
\hline SQ-LNS & SAA1 & 36468.54807 & 159310.3319 \\
\hline SQ-LNS & APOA & 7.498998449 & 9.154410747 \\
\hline SQ-LNS & HEMO & 7573.108655 & 10014.01744 \\
\hline SQ-LNS & APOC3 & 46608.03706 & 37034.22342 \\
\hline SQ-LNS & APOF & 1848.63341 & 1251.221326 \\
\hline SQ-LNS & HECF & 2476.216139 & 1802.009654 \\
\hline SQ-LNS & KNG1 & 7245.733778 & 9483.517209 \\
\hline SQ-LNS & APOH & 2032.34937 & 2009.99746 \\
\hline SQ-LNS & PON1 & 38.54713922 & 131.8124813 \\
\hline SQ-LNS & CLUS & 2777.09503 & 1152.797746 \\
\hline SQ-LNS & APOB & 4911.270736 & 2882.565501 \\
\hline SQ-LNS & PON3 & 751.040393 & 652.1189335 \\
\hline SQ-LNS & APOA5 & 11.42376376 & 11.22191583 \\
\hline
\end{tabular}




\begin{tabular}{|c|c|c|c|}
\hline Treatment & Glycopeptides ( $\mathrm{n}=163$ ) & $\begin{array}{l}\text { Mean } \\
\text { Abundance }\end{array}$ & SD \\
\hline IFA & APOB_3224_54020_z3 & 0.0394947 & 0.0172598 \\
\hline IFA & APOB_3411_5401 & 0.0128901 & 0.0227725 \\
\hline IFA & AACT_271_6512 & 0.0078521 & 0.0076865 \\
\hline IFA & AACT_271_7603 & 0.031695 & 0.0255303 \\
\hline IFA & AACT_271_7602 & 0.0482093 & 0.0290721 \\
\hline IFA & AACT_271_6502 & 0.0020941 & 0.0022993 \\
\hline IFA & AACT_106_7603 & 0.0021432 & 0.0015894 \\
\hline IFA & APOB_185_52000_z3 & 0.8218095 & 1.5675627 \\
\hline IFA & APOB_983_5401 & 0.0252867 & 0.0106358 \\
\hline IFA & AACT_106_7604 & 0.0005456 & 0.0006506 \\
\hline IFA & A1BG_179_5421/5402 & 0.0178749 & 0.0039714 \\
\hline IFA & APOB_983_54020_z4 & 0.0412918 & 0.0144838 \\
\hline IFA & AACT_127_5401 & 0.0018222 & 0.0013482 \\
\hline IFA & AACT_127_8500 & 0.0454916 & 0.052064 \\
\hline IFA & A1AT_271_5412 & 0.0005245 & 0.0004244 \\
\hline IFA & A1AT_271_5402 & 0.0527909 & 0.0206704 \\
\hline IFA & A1AT_271_55110_z3 & 0.0002886 & 0.0002232 \\
\hline IFA & A1AT_271_5401 & 0.0005451 & 0.0003676 \\
\hline IFA & A1AT_271_6503 & 0.0002403 & 0.0002031 \\
\hline IFA & APOA2_35_11020_z3 & 0.0010285 & 0.0008335 \\
\hline IFA & APOA2_35_11010_z2 & 0.0000227 & 0.0000456 \\
\hline IFA & A1AT_271MC_5412 & 0.005606 & 0.00441 \\
\hline IFA & A1AT_107_5401 & 0.0174233 & 0.0101624 \\
\hline IFA & A1AT_107_5411 & 0.0356425 & 0.009788 \\
\hline IFA & A1AT_271MC_5402 & 0.1437275 & 0.0444519 \\
\hline IFA & A1AT_107_6512 & 0.0103113 & 0.0077278 \\
\hline IFA & A1AT_107_6502/6521 & 0.0265805 & 0.0107613 \\
\hline IFA & A1AT_107_5402 & 0.0606979 & 0.0154517 \\
\hline IFA & A1AT_107_5412 & 0.0051522 & 0.0021896 \\
\hline IFA & A1AT_107_6513 & 0.0084881 & 0.0058065 \\
\hline IFA & A1AT_107_6503 & 0.0256588 & 0.0102744 \\
\hline IFA & A1AT_107_6501/6520 & 0.0020917 & 0.0050324 \\
\hline IFA & APOA2_95_11010_z2 & 0.0003368 & 0.0001962 \\
\hline IFA & APOA2_88_11010_z2 & 0.0002829 & 0.0001727 \\
\hline IFA & A1AT_70_5412 & 0.0008311 & 0.0003813 \\
\hline IFA & A1AT_70_5402 & 0.0503268 & 0.0193743 \\
\hline IFA & APOD_65_65210_z3 & 0.0000209 & 0.0000178 \\
\hline IFA & APOD_65_65030_z3 & 0.000045 & 0.000044 \\
\hline IFA & APOD_98_54100_z3 & 0.0013688 & 0.0015123 \\
\hline IFA & APOD_98_54000_z3 & 0.0001259 & 0.0000645 \\
\hline
\end{tabular}




\begin{tabular}{|c|c|c|c|}
\hline IFA & APOD_65_65020_z3 & 0.0000239 & 0.0000288 \\
\hline IFA & APOD_65_65010_z3 & 0.0000257 & 0.000018 \\
\hline IFA & APOD_65_65020_z3_deamidated & 0.0001719 & 0.0001575 \\
\hline IFA & APOD_98_65010_z3 & 0.0000192 & 0.0000233 \\
\hline IFA & APOD_98_65110_z3 & 0.000041 & 0.0000257 \\
\hline IFA & APOD_65_54010_z3 & 0.0000572 & 0.0000369 \\
\hline IFA & APOD_98_54110_z3 & 0.000161 & 0.0000881 \\
\hline IFA & APOD_98_54020_z3 & 0.0000193 & 0.0000221 \\
\hline IFA & APOD_98_54010_z3 & 0.0000907 & 0.0000686 \\
\hline IFA & APOD_65_54020_z3 & 0.000032 & 0.0000238 \\
\hline IFA & APOD_98_65130_z3 & 0.0000244 & 0.0000252 \\
\hline IFA & APOD_98_54120_z3 & 0.0000332 & 0.00003 \\
\hline IFA & APOD_65_76020_z3 & 0.0000297 & 0.000028 \\
\hline IFA & APOD_98_5402 & 0.0004665 & 0.0002325 \\
\hline IFA & APOD_98_65320_z3 & 0.0000072 & 0.0000077 \\
\hline IFA & APOD_65_54200_z3 & 0.0000498 & 0.0000387 \\
\hline IFA & APOD_65_76210_z3 & 0.0000263 & 0.0000226 \\
\hline IFA & APOD_65_65030_z3_deamidated & 0.0000185 & 0.0000137 \\
\hline IFA & APOD_98_6520 & 0.0002007 & 0.0001675 \\
\hline IFA & APOD_98_6510 & 0.0005036 & 0.0004456 \\
\hline IFA & APOD_98_9800 & 0.0003908 & 0.0006734 \\
\hline IFA & APOD_98_6530 & 0.0006519 & 0.0005456 \\
\hline IFA & APOD_98_7600 & 0.0002658 & 0.0002406 \\
\hline IFA & APOC3_94AMC_12020_z3 & 0.0256366 & 0.0418916 \\
\hline IFA & APOC3_94AMC_22100_z3 & 0.0008421 & 0.0013659 \\
\hline IFA & APOC3_94_1210 & 0.0011263 & 0.0014675 \\
\hline IFA & APOC3_94_0310 & 0.0007523 & 0.0008252 \\
\hline IFA & APOC3_94_0300 & 0.1284821 & 0.1720296 \\
\hline IFA & APOC3_94AMC_11000_z3 & 0.0048801 & 0.0022002 \\
\hline IFA & APOC3_94MC_11010_z3 & 0.0273929 & 0.0125607 \\
\hline IFA & APOC3_94A_11010_z2 & 0.0010305 & 0.0008245 \\
\hline IFA & APOC3_94MC_1102 & 0.1616978 & 0.031748 \\
\hline IFA & APOC3_94MC_11020_z3 & 0.0112174 & 0.0057145 \\
\hline IFA & APOC3_94_1101 & 0.1479717 & 0.0421585 \\
\hline IFA & APOC3_94A_11000_z2 & 0.0006624 & 0.0005272 \\
\hline IFA & AP0C3_94Aoff_1101 & 0.0080437 & 0.0048694 \\
\hline IFA & APOC3_94_2110 & 0.0009794 & 0.0012795 \\
\hline IFA & APOC3_94Aoff_1102 & 0.0056151 & 0.0045721 \\
\hline IFA & APOC3_94_1102 & 0.1055842 & 0.023329 \\
\hline IFA & APOC3_94A_11020_z2 & 0.0002285 & 0.000188 \\
\hline IFA & APOC3_94_1111 & 0.0056157 & 0.0138182 \\
\hline IFA & APOC3_94_1300 & 0.0030746 & 0.0043121 \\
\hline IFA & APOC3_94_1202 & 0.0057645 & 0.0081593 \\
\hline
\end{tabular}




\begin{tabular}{|c|c|c|c|}
\hline IFA & CLUS_291_5400 & 0.0573338 & 0.0744249 \\
\hline IFA & CLUS_291_6503 & 0.018689 & 0.0390782 \\
\hline IFA & CLUS_291_54020_z3 & 0.1482094 & 0.0418989 \\
\hline IFA & CLUS_291_5421/5402 & 0.1232955 & 0.0391082 \\
\hline IFA & CLUS_291_5420/5401 & 0.0408822 & 0.0241741 \\
\hline IFA & CLUS_86_6503 & 0.0013877 & 0.0007987 \\
\hline IFA & APOF_269_11010_z2 & 0.0808883 & 0.0227037 \\
\hline IFA & APOF_273/274_11010_z3 & 2.251669 & 0.4186461 \\
\hline IFA & APOF_273/274_11020_z3 & 0.9569323 & 0.1903533 \\
\hline IFA & APOE_307/308_11010_z3 & 0.038893 & 0.0090965 \\
\hline IFA & APOE_307/308_11010_z2 & 0.0010153 & 0.0003066 \\
\hline IFA & CLUS_374_6520/6501 & 0.1244688 & 0.0534979 \\
\hline IFA & CLUS_374_5402/5421 & 0.106659 & 0.0333114 \\
\hline IFA & APOE_215_11010_z2 & 0.0062528 & 0.0067569 \\
\hline IFA & CLUS_374_5420/5401 & 1.7521068 & 1.1364774 \\
\hline IFA & APOE_215_11020_z2 & 0.001846 & 0.0016198 \\
\hline IFA & CLUS_374_6512 & 0.1395672 & 0.0940911 \\
\hline IFA & C03_85_6200 & 0.5173679 & 0.2220976 \\
\hline IFA & C03_85_5200 & 0.4647495 & 0.218606 \\
\hline IFA & C03_85_7200 & 0.2139657 & 0.1496251 \\
\hline IFA & APOM_135_43010_z3 & 0.0006191 & 0.0002381 \\
\hline IFA & APOM_135_54010_z3 & 0.00039 & 0.0000915 \\
\hline IFA & APOM_135_53010_z3 & 0.0001145 & 0.0000548 \\
\hline IFA & C03_85_6301 & 1.0192401 & 0.7802088 \\
\hline IFA & APOM_135_54020_z3 & 0.000154 & 0.0000673 \\
\hline IFA & APOM_135_44010_z3 & 0.0000445 & 0.0000384 \\
\hline IFA & APOM_135_55110_z3 & 0.0000304 & 0.0000166 \\
\hline IFA & C1S_174_5402 & 0.0083218 & 0.0048297 \\
\hline IFA & C1S_174_5401 & 0.0092951 & 0.0068835 \\
\hline IFA & APOM_135_5421 & 0.0001529 & 0.0001102 \\
\hline IFA & APOM_135_8500 & 0.0000314 & 0.0000162 \\
\hline IFA & HEMO_240/246_5402 & 0.0015473 & 0.0016394 \\
\hline IFA & HEMO_187_6503 & 0.0186528 & 0.005605 \\
\hline IFA & HEMO_187_5402/5421 & 0.0904443 & 0.012613 \\
\hline IFA & HEMO_187_5412/5431 & 0.0045839 & 0.0029389 \\
\hline IFA & FETUA_346_1101 & 0.644887 & 0.1071992 \\
\hline IFA & FETUA_346_2200 & 0.0021247 & 0.0012919 \\
\hline IFA & FETUA_156_6411 & 0.0020513 & 0.0016073 \\
\hline IFA & FETUA_156_5400 & 0.0080567 & 0.0056716 \\
\hline IFA & FETUA_156_5401 & 0.0370009 & 0.0111281 \\
\hline IFA & HEP2_49_5402 & 0.3926462 & 0.2115345 \\
\hline IFA & FETUA_156_6410 & 0.0150072 & 0.0089408 \\
\hline IFA & FETUA_156_6502 & 0.0262995 & 0.0097264 \\
\hline
\end{tabular}




\begin{tabular}{|c|c|c|c|}
\hline IFA & FETUA_156_5402/5421 & 0.4521056 & 0.0788971 \\
\hline IFA & FETUA_156_6503 & 0.0258202 & 0.0103454 \\
\hline IFA & FETUA_176_6501 & 0.0272377 & 0.0176713 \\
\hline IFA & HEP2_49_5412 & 0.019682 & 0.0158072 \\
\hline IFA & FETUA_156_6513 & 0.003414 & 0.0030239 \\
\hline IFA & HEMO_453_5420/5401 & 0.148944 & 0.0538416 \\
\hline IFA & FETUA_176_5401 & 0.0883577 & 0.033112 \\
\hline IFA & FETUA_176_6512 & 0.0063118 & 0.0036247 \\
\hline IFA & FETUA_176_6502 & 0.0114561 & 0.0050218 \\
\hline IFA & FETUA_176_6513 & 0.004584 & 0.0027493 \\
\hline IFA & FETUA_176_7600 & 0.0415096 & 0.0111661 \\
\hline IFA & FETUA_176_5412/5431 & 0.0213714 & 0.0057454 \\
\hline IFA & FETUA_176_5402/5421 & 0.1178337 & 0.0147793 \\
\hline IFA & FETUA_176_6503 & 0.0083317 & 0.00254 \\
\hline IFA & HEMO_453_5402/5421 & 0.1625453 & 0.0366154 \\
\hline IFA & LCAT_108_65030_z3 & 0.0146358 & 0.0045639 \\
\hline IFA & KNG1_205_6503 & 0.006725 & 0.0035589 \\
\hline IFA & KNG1_205_5402/5421 & 0.0035403 & 0.0019222 \\
\hline IFA & KNG1_205_6512 & 0.0069067 & 0.0081522 \\
\hline IFA & PON3_29_76030/76220_z3 & 0.2808948 & 0.491807 \\
\hline IFA & PON1_253_5402/5421 & 25.6158152 & 30.4351972 \\
\hline IFA & PON1_253_4301 & 21.5265258 & 29.3755552 \\
\hline IFA & PON1_253_54020_z3 & 12.2262258 & 18.6821024 \\
\hline IFA & PON1_253_43010_z3 & 184.797944 & 227.7171975 \\
\hline IFA & PON3_29_75310_z3 & 3.0430789 & 5.8493337 \\
\hline IFA & PON3_29_76210_z3 & 4.6268155 & 10.2021441 \\
\hline IFA & PON1_324_65030_z3 & 0.4699565 & 0.7920262 \\
\hline IFA & KNG1_169_5402 & 0.0027837 & 0.0033689 \\
\hline IFA & PON1_324_6502 & 49.4403839 & 63.8274665 \\
\hline IFA & PON1_324_5420 & 0.8756779 & 2.0658797 \\
\hline IFA & PON1_324_6501 & 3.9249468 & 6.3021571 \\
\hline IFA & SAA_94_55100_z3 & 0.0016891 & 0.0024032 \\
\hline IFA & SAA_94_54010_z3 & 0.007496 & 0.0089788 \\
\hline IFA & SAA_94_44010_z3 & 0.000459 & 0.0007009 \\
\hline IFA & SAA_94_53010_z3 & 0.0004467 & 0.0004924 \\
\hline IFA & SAA_94_43010_z3 & 0.0019437 & 0.0037609 \\
\hline IFA & SAA_94_54020_z3 & 0.0024335 & 0.004961 \\
\hline SQ-LNS & APOB_3224_54020_z3 & 0.0360742 & 0.0205106 \\
\hline SQ-LNS & APOB_3411_5401 & 0.0133048 & 0.031548 \\
\hline SQ-LNS & AACT_271_6512 & 0.0092851 & 0.0111823 \\
\hline SQ-LNS & AACT_271_7603 & 0.0433408 & 0.0328622 \\
\hline SQ-LNS & AACT_271_7602 & 0.0565462 & 0.0307353 \\
\hline SQ-LNS & AACT_271_6502 & 0.0037181 & 0.0036589 \\
\hline
\end{tabular}




\begin{tabular}{|c|c|c|c|}
\hline SQ-LNS & AACT_106_7603 & 0.0030077 & 0.0025984 \\
\hline SQ-LNS & APOB_185_52000_z3 & 0.5967682 & 0.886622 \\
\hline SQ-LNS & APOB_983_5401 & 0.0238097 & 0.013511 \\
\hline SQ-LNS & AACT_106_7604 & 0.0006532 & 0.0010299 \\
\hline SQ-LNS & A1BG_179_5421/5402 & 0.0187236 & 0.0051327 \\
\hline SQ-LNS & APOB_983_54020_z4 & 0.042181 & 0.0188127 \\
\hline SQ-LNS & AACT_127_5401 & 0.0019927 & 0.0020864 \\
\hline SQ-LNS & AACT_127_8500 & 0.0503658 & 0.0478195 \\
\hline SQ-LNS & A1AT_271_5412 & 0.0005837 & 0.0004937 \\
\hline SQ-LNS & A1AT_271_5402 & 0.066594 & 0.0372713 \\
\hline SQ-LNS & A1AT_271_55110_z3 & 0.0004226 & 0.000366 \\
\hline SQ-LNS & A1AT_271_5401 & 0.0006625 & 0.0005339 \\
\hline SQ-LNS & A1AT_271_6503 & 0.000254 & 0.0002328 \\
\hline SQ-LNS & APOA2_35_11020_z3 & 0.0014228 & 0.0012092 \\
\hline SQ-LNS & APOA2_35_11010_z2 & 0.0000171 & 0.0000361 \\
\hline SQ-LNS & A1AT_271MC_5412 & 0.0045508 & 0.0033261 \\
\hline SQ-LNS & A1AT_107_5401 & 0.0199933 & 0.013445 \\
\hline SQ-LNS & A1AT_107_5411 & 0.0384509 & 0.0131449 \\
\hline SQ-LNS & A1AT_271MC_5402 & 0.1519575 & 0.0496907 \\
\hline SQ-LNS & A1AT_107_6512 & 0.011594 & 0.0086922 \\
\hline SQ-LNS & A1AT_107_6502/6521 & 0.031491 & 0.0203581 \\
\hline SQ-LNS & A1AT_107_5402 & 0.0766724 & 0.0333366 \\
\hline SQ-LNS & A1AT_107_5412 & 0.0060377 & 0.0027078 \\
\hline SQ-LNS & A1AT_107_6513 & 0.010431 & 0.0068266 \\
\hline SQ-LNS & A1AT_107_6503 & 0.0316656 & 0.0147775 \\
\hline SQ-LNS & A1AT_107_6501/6520 & 0.0030173 & 0.0066414 \\
\hline SQ-LNS & APOA2_95_11010_z2 & 0.00039 & 0.0002525 \\
\hline SQ-LNS & APOA2_88_11010_z2 & 0.0003228 & 0.0002112 \\
\hline SQ-LNS & A1AT_70_5412 & 0.0008328 & 0.0004879 \\
\hline SQ-LNS & A1AT_70_5402 & 0.0708743 & 0.0403989 \\
\hline SQ-LNS & APOD_65_65210_z3 & 0.0000199 & 0.0000288 \\
\hline SQ-LNS & APOD_65_65030_z3 & 0.0000452 & 0.0000455 \\
\hline SQ-LNS & APOD_98_54100_z3 & 0.0011974 & 0.0018509 \\
\hline SQ-LNS & APOD_98_54000_z3 & 0.0001162 & 0.0000727 \\
\hline SQ-LNS & APOD_65_65020_z3 & 0.0000194 & 0.0000217 \\
\hline SQ-LNS & APOD_65_65010_z3 & 0.0000266 & 0.0000301 \\
\hline SQ-LNS & APOD_65_65020_z3_deamidated & 0.0002 & 0.0002207 \\
\hline SQ-LNS & APOD_98_65010_z3 & 0.0000155 & 0.0000134 \\
\hline SQ-LNS & APOD_98_65110_z3 & 0.0000361 & 0.0000263 \\
\hline SQ-LNS & APOD_65_54010_z3 & 0.0000462 & 0.0000327 \\
\hline SQ-LNS & APOD_98_54110_z3 & 0.0002055 & 0.0001553 \\
\hline SQ-LNS & APOD_98_54020_z3 & 0.0000174 & 0.0000198 \\
\hline SQ-LNS & APOD_98_54010_z3 & 0.0000901 & 0.0000841 \\
\hline
\end{tabular}




\begin{tabular}{|c|c|c|c|}
\hline SQ-LNS & APOD_65_54020_z3 & 0.000037 & 0.000028 \\
\hline SQ-LNS & APOD_98_65130_z3 & 0.0000195 & 0.000026 \\
\hline SQ-LNS & APOD_98_54120_z3 & 0.0000516 & 0.0000964 \\
\hline SQ-LNS & APOD_65_76020_z3 & 0.0000268 & 0.0000183 \\
\hline SQ-LNS & APOD_98_5402 & 0.0005348 & 0.0005597 \\
\hline SQ-LNS & APOD_98_65320_z3 & 0.0000097 & 0.0000116 \\
\hline SQ-LNS & APOD_65_54200_z3 & 0.000054 & 0.0000444 \\
\hline SQ-LNS & APOD_65_76210_z3 & 0.0000289 & 0.0000282 \\
\hline SQ-LNS & APOD_65_65030_z3_deamidated & 0.0000229 & 0.0000234 \\
\hline SQ-LNS & APOD_98_6520 & 0.0001724 & 0.0001188 \\
\hline SQ-LNS & APOD_98_6510 & 0.0003984 & 0.0003359 \\
\hline SQ-LNS & APOD_98_9800 & 0.0004614 & 0.0012463 \\
\hline SQ-LNS & APOD_98_6530 & 0.0005245 & 0.0005251 \\
\hline SQ-LNS & APOD_98_7600 & 0.0002207 & 0.0002143 \\
\hline SQ-LNS & APOC3_94AMC_12020_z3 & 0.0179115 & 0.038897 \\
\hline SQ-LNS & APOC3_94AMC_22100_z3 & 0.0007987 & 0.0010032 \\
\hline SQ-LNS & APOC3_94_1210 & 0.00149 & 0.0039736 \\
\hline SQ-LNS & APOC3_94_0310 & 0.0009661 & 0.0016544 \\
\hline SQ-LNS & APOC3_94_0300 & 0.0925521 & 0.0234368 \\
\hline SQ-LNS & APOC3_94AMC_11000_z3 & 0.0043751 & 0.0017712 \\
\hline SQ-LNS & AP0C3_94MC_11010_z3 & 0.0230502 & 0.0090266 \\
\hline SQ-LNS & APOC3_94A_11010_z2 & 0.0009175 & 0.0004671 \\
\hline SQ-LNS & AP0C3_94MC_1102 & 0.1548663 & 0.0333214 \\
\hline SQ-LNS & AP0C3_94MC_11020_z3 & 0.0102879 & 0.0059773 \\
\hline SQ-LNS & APOC3_94_1101 & 0.1279492 & 0.0439271 \\
\hline SQ-LNS & APOC3_94A_11000_z2 & 0.0006756 & 0.0006089 \\
\hline SQ-LNS & APOC3_94Aoff_1101 & 0.0077636 & 0.0036551 \\
\hline SQ-LNS & APOC3_94_2110 & 0.0006797 & 0.0013118 \\
\hline SQ-LNS & APOC3_94Aoff_1102 & 0.0058798 & 0.0057642 \\
\hline SQ-LNS & APOC3_94_1102 & 0.0984933 & 0.0272781 \\
\hline SQ-LNS & APOC3_94A_11020_z2 & 0.0002145 & 0.000195 \\
\hline SQ-LNS & APOC3_94_1111 & 0.0023424 & 0.0042113 \\
\hline SQ-LNS & APOC3_94_1300 & 0.001765 & 0.0026773 \\
\hline SQ-LNS & APOC3_94_1202 & 0.0051276 & 0.011014 \\
\hline SQ-LNS & CLUS_291_5400 & 0.035016 & 0.0279911 \\
\hline SQ-LNS & CLUS_291_6503 & 0.0125713 & 0.0202466 \\
\hline SQ-LNS & CLUS_291_54020_z3 & 0.1369051 & 0.0434454 \\
\hline SQ-LNS & CLUS_291_5421/5402 & 0.104943 & 0.0327815 \\
\hline SQ-LNS & CLUS_291_5420/5401 & 0.0346106 & 0.0154522 \\
\hline SQ-LNS & CLUS_86_6503 & 0.001432 & 0.0012173 \\
\hline SQ-LNS & APOF_269_11010_z2 & 0.0911866 & 0.0614788 \\
\hline SQ-LNS & APOF_273/274_11010_z3 & 2.2598937 & 0.5417472 \\
\hline SQ-LNS & APOF_273/274_11020_z3 & 0.9932302 & 0.2866299 \\
\hline
\end{tabular}




\begin{tabular}{|c|c|c|c|}
\hline SQ-LNS & APOE_307/308_11010_z3 & 0.0397624 & 0.0098877 \\
\hline SQ-LNS & APOE_307/308_11010_z2 & 0.0011287 & 0.0003328 \\
\hline SQ-LNS & CLUS_374_6520/6501 & 0.104148 & 0.0551642 \\
\hline SQ-LNS & CLUS_374_5402/5421 & 0.10461 & 0.0369595 \\
\hline SQ-LNS & APOE_215_11010_z2 & 0.0050296 & 0.0048735 \\
\hline SQ-LNS & CLUS_374_5420/5401 & 1.3892337 & 0.6961809 \\
\hline SQ-LNS & APOE_215_11020_z2 & 0.0016964 & 0.0019215 \\
\hline SQ-LNS & CLUS_374_6512 & 0.108697 & 0.0527778 \\
\hline SQ-LNS & C03_85_6200 & 0.4928755 & 0.3060726 \\
\hline SQ-LNS & C03_85_5200 & 0.4637935 & 0.241497 \\
\hline SQ-LNS & C03_85_7200 & 0.1941483 & 0.1536028 \\
\hline SQ-LNS & APOM_135_43010_z3 & 0.0006842 & 0.0002434 \\
\hline SQ-LNS & APOM_135_54010_z3 & 0.0004338 & 0.0001095 \\
\hline SQ-LNS & APOM_135_53010_z3 & 0.0001299 & 0.0000675 \\
\hline SQ-LNS & C03_85_6301 & 1.2139166 & 1.3940368 \\
\hline SQ-LNS & APOM_135_54020_z3 & 0.0001889 & 0.0000913 \\
\hline SQ-LNS & APOM_135_44010_z3 & 0.0000361 & 0.0000243 \\
\hline SQ-LNS & APOM_135_55110_z3 & 0.0000398 & 0.0000249 \\
\hline SQ-LNS & C1S_174_5402 & 0.0105521 & 0.0069264 \\
\hline SQ-LNS & C1S_174_5401 & 0.0164874 & 0.0365968 \\
\hline SQ-LNS & APOM_135_5421 & 0.0001364 & 0.00015 \\
\hline SQ-LNS & APOM_135_8500 & 0.0000353 & 0.0000281 \\
\hline SQ-LNS & HEMO_240/246_5402 & 0.0016205 & 0.0017465 \\
\hline SQ-LNS & HEMO_187_6503 & 0.0192163 & 0.008273 \\
\hline SQ-LNS & HEMO_187_5402/5421 & 0.0917258 & 0.0185436 \\
\hline SQ-LNS & HEMO_187_5412/5431 & 0.0041695 & 0.0025208 \\
\hline SQ-LNS & FETUA_346_1101 & 0.6826585 & 0.1214871 \\
\hline SQ-LNS & FETUA_346_2200 & 0.0032741 & 0.0022572 \\
\hline SQ-LNS & FETUA_156_6411 & 0.0030369 & 0.0025073 \\
\hline SQ-LNS & FETUA_156_5400 & 0.0080833 & 0.0060188 \\
\hline SQ-LNS & FETUA_156_5401 & 0.0379221 & 0.0163555 \\
\hline SQ-LNS & HEP2_49_5402 & 0.3712908 & 0.2009065 \\
\hline SQ-LNS & FETUA_156_6410 & 0.0223969 & 0.0145957 \\
\hline SQ-LNS & FETUA_156_6502 & 0.0285029 & 0.0151165 \\
\hline SQ-LNS & FETUA_156_5402/5421 & 0.4969865 & 0.1013284 \\
\hline SQ-LNS & FETUA_156_6503 & 0.0296815 & 0.0133201 \\
\hline SQ-LNS & FETUA_176_6501 & 0.0427938 & 0.0269402 \\
\hline SQ-LNS & HEP2_49_5412 & 0.0324887 & 0.0298518 \\
\hline SQ-LNS & FETUA_156_6513 & 0.005065 & 0.0039551 \\
\hline SQ-LNS & HEMO_453_5420/5401 & 0.139898 & 0.0651389 \\
\hline SQ-LNS & FETUA_176_5401 & 0.0796742 & 0.0377109 \\
\hline SQ-LNS & FETUA_176_6512 & 0.0063726 & 0.0044113 \\
\hline SQ-LNS & FETUA_176_6502 & 0.0115723 & 0.0068503 \\
\hline
\end{tabular}




\begin{tabular}{|l|l|r|r|} 
SQ-LNS & FETUA_176_6513 & 0.0048559 & 0.0024456 \\
\hline SQ-LNS & FETUA_176_7600 & 0.0401939 & 0.0122964 \\
\hline SQ-LNS & FETUA_176_5412/5431 & 0.0208814 & 0.0069375 \\
\hline SQ-LNS & FETUA_176_5402/5421 & 0.1181136 & 0.0198114 \\
\hline SQ-LNS & FETUA_176_6503 & 0.0084927 & 0.0030904 \\
\hline SQ-LNS & HEM0_453_5402/5421 & 0.1609827 & 0.0477325 \\
\hline SQ-LNS & LCAT_108_65030_z3 & 0.0154938 & 0.0044167 \\
\hline SQ-LNS & KNG1_205_6503 & 0.0085932 & 0.0062304 \\
\hline SQ-LNS & KNG1_205_5402/5421 & 0.0049045 & 0.003644 \\
\hline SQ-LNS & KNG1_205_6512 & 0.007613 & 0.0088827 \\
\hline SQ-LNS & PON3_29_76030/76220_z3 & 0.1891634 & 0.2198464 \\
\hline SQ-LNS & PON1_253_5402/5421 & 22.9291195 & 22.2153279 \\
\hline SQ-LNS & PON1_253_4301 & 17.7064821 & 21.8488609 \\
\hline SQ-LNS & PON1_253_54020_z3 & 9.8524447 & 10.2595725 \\
\hline SQ-LNS & PON1_253_43010_z3 & 180.4528761 & 223.2800303 \\
\hline SQ-LNS & PON3_29_75310_z3 & 2.0986005 & 2.6136493 \\
\hline SQ-LNS & PON3_29_76210_z3 & 2.9688579 & 4.9107168 \\
\hline SQ-LNS & PON1_324_65030_z3 & 0.4423539 & 0.5318954 \\
\hline SQ-LNS & KNG1_169_5402 & 0.004139 & 0.006088 \\
\hline SQ-LNS & PON1_324_6502 & 44.6511212 & 61.8072691 \\
\hline SQ-LNS & PON1_324_5420 & 0.718557 & 1.1075797 \\
\hline SQ-LNS & PON1_324_6501 & 3.1246833 & 4.6988605 \\
\hline SQ-LNS & SAA_94_55100_z3 & 0.0014148 & 0.0013801 \\
\hline SQ-LNS & SAA_94_54010_z3 & 0.0061682 & 0.0056536 \\
\hline SQ-LNS & SAA_94_44010_z3 & 0.0003726 & 0.0004328 \\
\hline SQ-LNS & SAA_94_53010_z3 & 0.0005309 & 0.0005368 \\
\hline SQ-LNS & SAA_94_43010_z3 & 0.0012074 & 0.0012706 \\
\hline SQ-LNS & SAA_94_54020_z3 & 0.0020447 & 0.0020592 \\
\hline
\end{tabular}




\begin{tabular}{|c|c|c|c|c|c|c|}
\hline Protein & $\mathbf{N}$ & $\mathbf{m}$ & $\begin{array}{l}\text { Number } \\
\text { Glycopeptide } \\
\text { Increased in } \\
\text { SQ-LNS }\end{array}$ & $\begin{array}{l}\text { Number } \\
\text { Glycopeptide } \\
\text { Decreased in } \\
\text { SQ-LNS }\end{array}$ & $\begin{array}{l}\text { P value } \\
\text { Increased }\end{array}$ & $\begin{array}{l}\text { P value } \\
\text { Decreased }\end{array}$ \\
\hline A1AT & 163 & 18 & 17 & 1 & 0.0001244 & 1 \\
\hline FETUA & 163 & 19 & 16 & 3 & 0.00393 & 0.9994 \\
\hline AACT & 163 & 8 & 8 & 0 & 0.006192 & 1 \\
\hline KNG1 & 163 & 4 & 4 & 0 & 0.08228 & 1 \\
\hline APOF & 163 & 3 & 3 & 0 & 0.1549 & 1 \\
\hline APOM & 163 & 8 & 6 & 2 & 0.1969 & 0.9479 \\
\hline C1S & 163 & 2 & 2 & 0 & 0.2899 & 1 \\
\hline APOA2 & 163 & 4 & 3 & 1 & 0.3727 & 0.9177 \\
\hline$A 1 B G$ & 163 & 1 & 1 & 0 & 0.5399 & 1 \\
\hline LCAT & 163 & 1 & 1 & 0 & 0.5399 & 1 \\
\hline HEMO & 163 & 6 & 3 & 3 & 0.7315 & 0.5807 \\
\hline APOE & 163 & 4 & 2 & 2 & 0.7475 & 0.6273 \\
\hline HEP2 & 163 & 2 & 1 & 1 & 0.7898 & 0.7101 \\
\hline APOB & 163 & 5 & 2 & 3 & 0.8622 & 0.4246 \\
\hline APOD & 163 & 27 & 12 & 15 & 0.9032 & 0.1899 \\
\hline $\mathrm{CO} 3$ & 163 & 4 & 1 & 3 & 0.9571 & 0.2525 \\
\hline SAA & 163 & 6 & 1 & 5 & 0.9915 & 0.07248 \\
\hline CLUS & 163 & 10 & 1 & 9 & 0.9997 & 0.004316 \\
\hline APOC3 & 163 & 20 & 4 & 16 & 0.9998 & 0.001103 \\
\hline PON3 & 163 & 3 & 0 & 3 & 1 & 0.0953 \\
\hline PON1 & 163 & 8 & 0 & 8 & 1 & 0.001625 \\
\hline
\end{tabular}

The enrichment analysis is done using the hypergeometric distribution. $\mathbf{N}$ is the total number of glycopeptides; $\mathbf{m}$ is the total number of glycopeptides of a particular protein; Number Glycopeptide Increase in SQ-LNS is the number of glycopeptides that are increased in the SQLNS group; Number Glycopeptide Decrease in SQ-LNS is the total number of glycopeptides that are decreased in SQ-LNS group (higher in the IFA group); $\mathbf{P}$ value Increased can be interpreted as the probability that having $x$ number of glycopeptides increased in the SQ-LNS group is by chance; $\mathbf{P}$ value Decreased can be interpreted as the probability that having $X$ number of glycopeptides decreased in the SQ-LNS group is by chance. SQ-LNS, small-quantity lipid-based nutrient supplements; IFA, iron and folic acid. 


\begin{tabular}{|c|c|c|c|c|}
\hline Predictor & Response & Model & $95 \% \mathrm{Cl}$ & Pval \\
\hline \multirow[t]{16}{*}{$\begin{array}{l}\text { Cholesterol Efflux } \\
\text { Capacity }\end{array}$} & \multirow[t]{2}{*}{ HCZ18 } & Unadjusted & $(-4.89 e-02,6.90 e-02)$ & 0.7358 \\
\hline & & Adjusted & $(-4.53 e-02,6.76 e-02)$ & 0.6957 \\
\hline & \multirow[t]{2}{*}{ HCZ1812 } & Unadjusted & $(-3.10 e-02,2.85 e-02)$ & 0.9345 \\
\hline & & Adjusted & $(-3.40 e-02,2.39 e-02)$ & 0.7289 \\
\hline & \multirow[t]{2}{*}{ LAZ18 } & Unadjusted & $(-8.91 e-02,3.30 e-02)$ & 0.3626 \\
\hline & & Adjusted & $(-8.84 e-02,3.47 e-02)$ & 0.3869 \\
\hline & \multirow[t]{2}{*}{ LAZ1812 } & Unadjusted & $(-1.22 e-02,3.95 e-02)$ & 0.2949 \\
\hline & & Adjusted & $(-2.10 e-02,2.62 e-02)$ & 0.8248 \\
\hline & \multirow[t]{2}{*}{ WAZ18 } & Unadjusted & $(-9.71 e-02,2.92 e-02)$ & 0.2884 \\
\hline & & Adjusted & $(-9.71 e-02,2.92 e-02)$ & 0.2884 \\
\hline & \multirow[t]{2}{*}{ WAZ1812 } & Unadjusted & $(-2.29 e-02,3.60 e-02)$ & 0.6567 \\
\hline & & Adjusted & $(-2.90 e-02,1.96 e-02)$ & 0.6988 \\
\hline & \multirow[t]{2}{*}{ WLZ18 } & Unadjusted & $(-9.10 e-02,3.60 e-02)$ & 0.3916 \\
\hline & & Adjusted & $(-6.90 e-02,6.35 e-02)$ & 0.9350 \\
\hline & \multirow[t]{2}{*}{ WLZ1812 } & Unadjusted & $(-3.46 e-02,3.71 e-02)$ & 0.9460 \\
\hline & & Adjusted & $(-3.64 e-02,2.17 e-02)$ & 0.6158 \\
\hline \multirow[t]{16}{*}{ Surface/core lipid ratio } & \multirow[t]{2}{*}{ HCZ18 } & Unadjusted & $(-4.72 e-01,7.25 e-01)$ & 0.6755 \\
\hline & & Adjusted & $(-4.85 e-01,6.72 e-01)$ & 0.7487 \\
\hline & \multirow[t]{2}{*}{ HCZ1812 } & Unadjusted & $(-3.67 e-01,2.47 e-01)$ & 0.6973 \\
\hline & & Adjusted & $(-3.46 e-01,2.60 e-01)$ & 0.7774 \\
\hline & \multirow[t]{2}{*}{ LAZ18 } & Unadjusted & $(-1.06 e+00,1.77 e-01)$ & 0.1598 \\
\hline & & Adjusted & $(-1.03 e+00,2.10 e-01)$ & 0.1921 \\
\hline & \multirow[t]{2}{*}{ LAZ1812 } & Unadjusted & $(-3.57 e-01,1.80 e-01)$ & 0.5124 \\
\hline & & Adjusted & $(-3.43 e-01,1.34 e-01)$ & 0.3845 \\
\hline & \multirow[t]{2}{*}{ WAZ18 } & Unadjusted & $(-1.04 e+00,2.43 e-01)$ & 0.2194 \\
\hline & & Adjusted & $(-1.04 e+00,2.43 e-01)$ & 0.2194 \\
\hline & \multirow[t]{2}{*}{ WAZ1812 } & Unadjusted & $(-5.06 e-01,9.57 e-02)$ & 0.1782 \\
\hline & & Adjusted & $(-4.37 e-01,5.36 e-02)$ & 0.1235 \\
\hline & \multirow[t]{2}{*}{ WLZ18 } & Unadjusted & $(-9.19 e-01,3.75 e-01)$ & 0.4054 \\
\hline & & Adjusted & $(-9.92 e-01,3.08 e-01)$ & 0.2975 \\
\hline & \multirow[t]{2}{*}{ WLZ1812 } & Unadjusted & $(-5.99 e-01,1.34 e-01)$ & 0.2094 \\
\hline & & Adjusted & $(-5.22 e-01,6.93 e-02)$ & 0.1311 \\
\hline \multirow[t]{9}{*}{$E O D_{18}$} & \multirow[t]{2}{*}{ HCZ18 } & Unadjusted & $(-1.86 e+00,2.56 e+00)$ & 0.7524 \\
\hline & & Adjusted & $(-2.02 e+00,2.21 e+00)$ & 0.9299 \\
\hline & \multirow[t]{2}{*}{ HCZ1812 } & Unadjusted & $(-9.23 e-02,2.16 e+00)$ & 0.0714 \\
\hline & & Adjusted & $(-3.90 e-01,1.91 e+00)$ & 0.1913 \\
\hline & \multirow[t]{2}{*}{ LAZ18 } & Unadjusted & $(-3.01 e+00,1.64 e+00)$ & 0.5600 \\
\hline & & Adjusted & $(-2.71 e+00,2.03 e+00)$ & 0.7776 \\
\hline & \multirow[t]{2}{*}{ LAZ1812 } & Unadjusted & $(-2.44 e-02,1.97 e+00)$ & 0.0557 \\
\hline & & Adjusted & $(3.86 e-02,1.83 e+00)$ & 0.0412 \\
\hline & WAZ18 & Unadjusted & $(-2.60 e+00,2.23 e+00)$ & 0.8784 \\
\hline
\end{tabular}




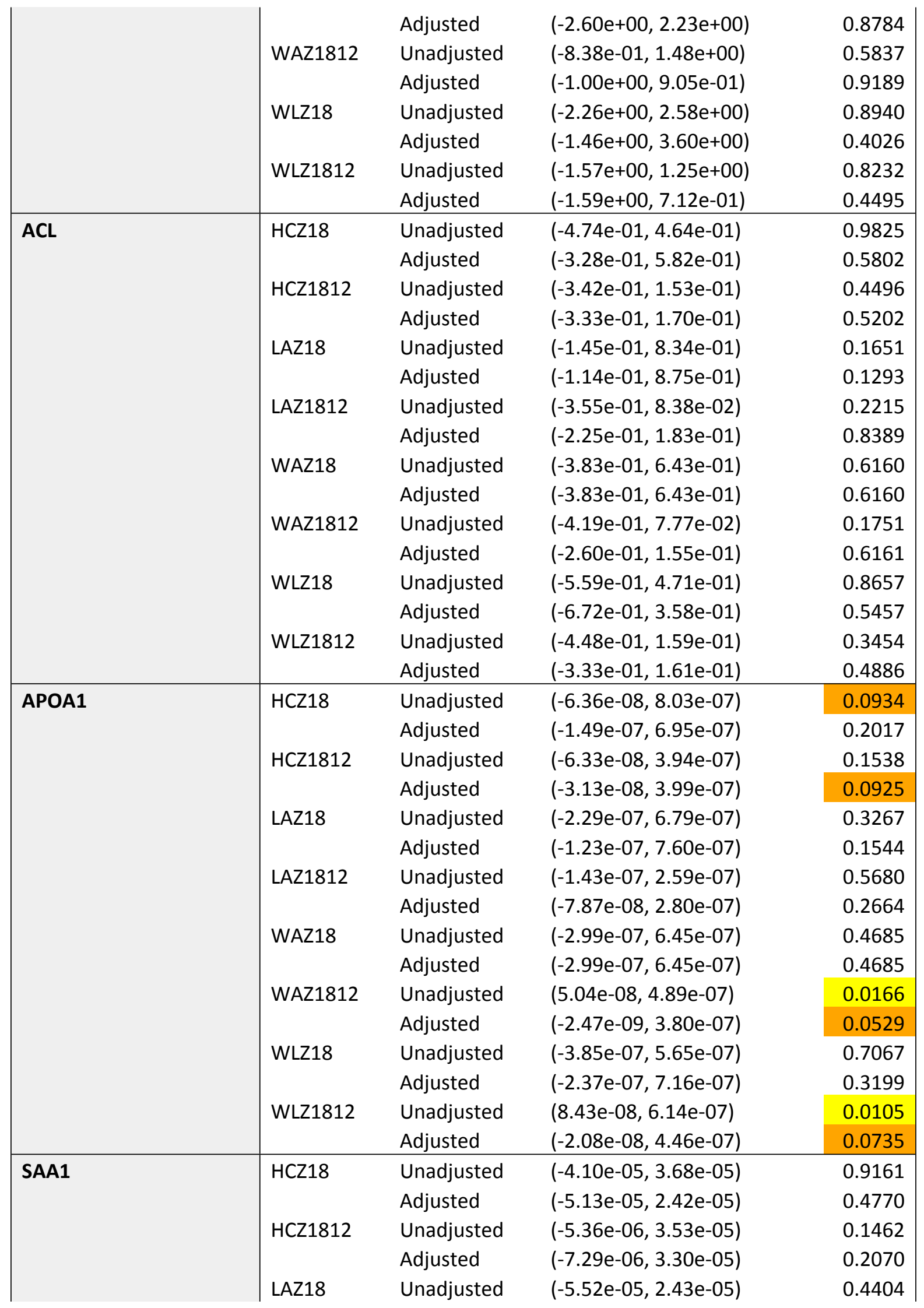




\begin{tabular}{|c|c|c|c|c|}
\hline & & Adjusted & $(-4.99 e-05,3.14 e-05)$ & 0.6524 \\
\hline & LAZ1812 & Unadjusted & $(-1.25 e-05,2.30 e-05)$ & 0.5547 \\
\hline & & Adjusted & $(-1.74 e-05,1.48 e-05)$ & 0.8703 \\
\hline & WAZ18 & Unadjusted & $(-5.74 e-05,2.49 e-05)$ & 0.4342 \\
\hline & & Adjusted & $(-5.74 e-05,2.49 e-05)$ & 0.4342 \\
\hline & WAZ1812 & Unadjusted & $(1.70 e-05,5.36 e-05)$ & 0.0003 \\
\hline & & Adjusted & $(5.13 e-06,3.88 e-05)$ & 0.0114 \\
\hline & WLZ18 & Unadjusted & $(-5.30 e-05,2.97 e-05)$ & 0.5770 \\
\hline & & Adjusted & $(-4.36 e-05,3.94 e-05)$ & 0.9208 \\
\hline & WLZ1812 & Unadjusted & $(2.53 e-05,6.88 e-05)$ & 0.0001 \\
\hline & & Adjusted & $(1.26 e-05,5.17 e-05)$ & 0.0017 \\
\hline SAA2 & HCZ18 & Unadjusted & $(-2.49 e-05,2.27 e-05)$ & 0.9242 \\
\hline & & Adjusted & $(-3.31 e-05,1.35 e-05)$ & 0.4060 \\
\hline & HCZ1812 & Unadjusted & $(-1.90 e-06,2.30 e-05)$ & 0.0953 \\
\hline & & Adjusted & $(-2.40 e-06,2.23 e-05)$ & 0.1123 \\
\hline & LAZ18 & Unadjusted & $(-3.24 e-05,1.60 e-05)$ & 0.5012 \\
\hline & & Adjusted & $(-3.13 e-05,1.76 e-05)$ & 0.5774 \\
\hline & LAZ1812 & Unadjusted & $(-8.29 e-06,1.34 e-05)$ & 0.6386 \\
\hline & & Adjusted & $(-9.72 e-06,9.74 e-06)$ & 0.9983 \\
\hline & WAZ18 & Unadjusted & $(-3.68 e-05,1.32 e-05)$ & 0.3507 \\
\hline & & Adjusted & $(-3.68 e-05,1.32 e-05)$ & 0.3507 \\
\hline & WAZ1812 & Unadjusted & $(1.16 e-05,3.37 e-05)$ & 0.0001 \\
\hline & & Adjusted & $(4.44 e-06,2.45 e-05)$ & 0.0054 \\
\hline & WLZ18 & Unadjusted & $(-3.56 e-05,1.47 e-05)$ & 0.4094 \\
\hline & & Adjusted & $(-2.91 e-05,2.17 e-05)$ & 0.7722 \\
\hline & WLZ1812 & Unadjusted & $(1.77 e-05,4.38 e-05)$ & 0.0000 \\
\hline & & Adjusted & $(8.88 e-06,3.27 e-05)$ & 0.0009 \\
\hline APOL1 & HCZ18 & Unadjusted & $(-3.19 e-05,2.56 e-05)$ & 0.8278 \\
\hline & & Adjusted & $(-3.56 e-05,1.96 e-05)$ & 0.5676 \\
\hline & HCZ1812 & Unadjusted & $(1.12 \mathrm{e}-06,2.98 \mathrm{e}-05)$ & 0.0350 \\
\hline & & Adjusted & $(-2.17 e-06,2.61 e-05)$ & 0.0957 \\
\hline & LAZ18 & Unadjusted & $(-4.23 e-05,1.81 e-05)$ & 0.4278 \\
\hline & & Adjusted & $(-3.74 e-05,2.27 e-05)$ & 0.6274 \\
\hline & LAZ1812 & Unadjusted & $(-8.61 e-06,1.77 e-05)$ & 0.4933 \\
\hline & & Adjusted & $(-1.23 e-05,1.16 e-05)$ & 0.9510 \\
\hline & WAZ18 & Unadjusted & $(-3.09 e-05,3.19 e-05)$ & 0.9754 \\
\hline & & Adjusted & $(-3.09 e-05,3.19 e-05)$ & 0.9754 \\
\hline & WAZ1812 & Unadjusted & $(1.21 e-05,3.93 e-05)$ & 0.0004 \\
\hline & & Adjusted & $(6.56 e-06,3.02 e-05)$ & 0.0028 \\
\hline & WLZ18 & Unadjusted & $(-2.24 e-05,4.05 e-05)$ & 0.5678 \\
\hline & & Adjusted & $(-2.00 e-05,4.26 e-05)$ & 0.4738 \\
\hline & WLZ1812 & Unadjusted & $(1.65 e-05,4.92 e-05)$ & 0.0002 \\
\hline & & Adjusted & $(1.23 e-05,3.97 e-05)$ & 0.0003 \\
\hline
\end{tabular}


ACL, average chain length; APOA1, apolipoprotein A-I; APOL1, apolipoprotein L1; CEC, cholesterol efflux capacity; EOD18, equivalent of double bond per 18 carbon; SAA1, serum amyloid A-1; SAA2, serum amyloid A-2; HCZ, head circumference for age Z-score; LAZ, length for age z-score; WAZ, weight for age z-score; WLZ, weight for length z-score; 18, at $18 \mathrm{mo}$; 1812 , changes in growth from 12 to $18 \mathrm{mo}$. 


\begin{tabular}{|l|l|l|}
\hline \multicolumn{3}{|l|}{ Table S6. Composition of supplements } \\
\hline Nutrient & IFA & SQ-LNS \\
\hline Ration, g/d & & 20 \\
\hline Total energy, kcal & & 118 \\
\hline Protein, g & & 2.6 \\
\hline Fat, g & & 10 \\
\hline Linoleic acid, g & & 4.59 \\
\hline$\alpha$-Linolenic acid, g & & 0.59 \\
\hline Vitamin A, $\mu$ RE & & 800 \\
\hline Vitamin C, mg & & 100 \\
\hline Vitamin B-1, mg & & 2.8 \\
\hline Vitamin B-2, mg & & 2.8 \\
\hline Niacin, mg & & 36 \\
\hline Folic acid, $\mu \mathrm{g}$ & 400 & 400 \\
\hline $\begin{array}{l}\text { Pantothenic acid, } \\
\text { mg }\end{array}$ & & 7 \\
\hline Vitamin B-6, mg & & 3.8 \\
\hline Vitamin B-12, $\mu \mathrm{g}$ & & 5.2 \\
\hline Vitamin D, IU & & 400 \\
\hline Vitamin E, mg & & 20 \\
\hline Vitamin K, $\mu$ g & & 45 \\
\hline Iron, mg & 60 & 20 \\
\hline Zinc, mg & & 30 \\
\hline Copper, mg & 4 \\
\hline Calcium, mg & 280 \\
\hline Phosphorus, mg & & 190 \\
\hline Potassium, mg & & 200 \\
\hline Magnesium, mg & & 65 \\
\hline Selenium, $\mu \mathrm{g}$ & & 130 \\
\hline Iodine, $\mu \mathrm{g}$ & & 250 \\
\hline Manganese, mg & 2.6 \\
\hline
\end{tabular}

IFA, iron and folic acid; SQ-LNS, small-quantity lipid-based nutrient supplements 


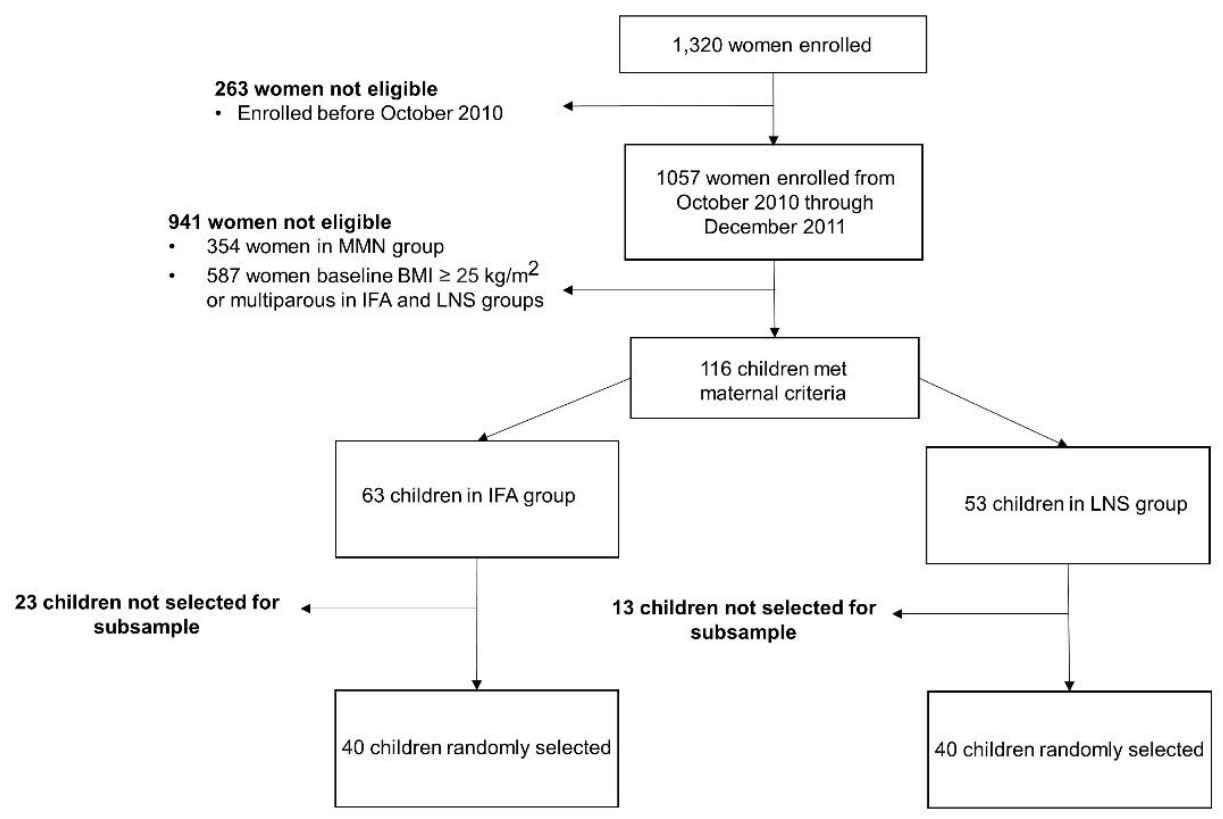

Supporting Information Figure S1. Flowchart of study profile 


\section{Supporting Information Method 1:}

\section{HDL Protein and Glycoprotein Identification}

Isolated HDL fractions were diluted in a total of $100 \mu \mathrm{L}$ with $50 \mathrm{mM}$ ammonium

bicarbonate buffer at $\mathrm{pH}$ 7.5. All reagents used for sample preparation were freshly prepared in a buffer of $50 \mathrm{mM}$ ammonium bicarbonate. Proteins were then denatured with $2 \mu \mathrm{L}$ of $550 \mathrm{mM}$ dithiothreitol (Promega, Madison, $\mathrm{WI}$ ) for $1 \mathrm{~h}$ at $65^{\circ} \mathrm{C}$ and alkylated with $4 \mu \mathrm{L}$ of $450 \mathrm{mM}$ iodoacetamide (Sigma-Aldrich, St. Louis, MO) for $30 \mathrm{~min}$ at room temperature away from light. Proteins were digested with $2 \mu \mathrm{g}$ sequencing grade trypsin (Promega) for $18 \mathrm{~h}$ at $37^{\circ} \mathrm{C}$. Samples were purified through Bond Elut C18 solid-phase extraction (Agilent, Santa Clara, CA), dried in a vacuum concentrator and reconstituted in $50 \mu \mathrm{L}$ LC-MS grade water.

Samples were run on an Agilent 1290 Infinity II High-Performance Liquid Chromatography (HPLC) coupled to a Fusion Lumos MS/MS Orbitrap (Thermo Fisher Scientific). The HPLC was equipped with a $150 \mathrm{~mm}$ Agilent Zorbax Eclipse Plus C18 column with $1.8 \mu \mathrm{m}$ particle size. Peptides and glycopeptides were eluted with a binary gradient of $(A) 3 \%$ acetonitrile with $0.1 \%$ formic acid in water, and (B) $90 \%$ acetonitrile with $0.1 \%$ formic acid in water. The HPLC was set at a flow rate of $0.3 \mathrm{~mL} / \mathrm{min}$ and programmed to ramp from $0 \%$ to $20 \% \mathrm{~B}$ in $20 \mathrm{~min}, 30 \%$ at 40 $\min , 44 \%$ at $47 \mathrm{~min}$, and $100 \%$ at $48 \mathrm{~min}$ followed by a flushing and equilibration cycle. The electrospray ionization (ESI) voltage was set to $3500 \mathrm{~V}$ in the positive mode.

The Orbitrap was operated in positive mode with a precursor scan resolution of 60,000 and a range of $350-2000 \mathrm{~m} / \mathrm{z}$. Fragmentation was accomplished by stepped High-energy Collisional Dissociation (HCD). The collision energy was set at $30 \%$ and stepped at $10 \%$. Ions for fragmentation were filtered to include a precursor mass range of $700-2000 \mathrm{~m} / \mathrm{z}$ and charge states between 2-6.

Peptides and glycopeptides were identified with Byonic software (Protein Metrics Inc) from the Orbitrap MS data. For glycopeptide identification, a database of protein sequences and a library of glycan compositions are required as inputs. The human proteome database was 
downloaded from Uniprot.org. We used in-house libraries for $\mathrm{N}$-glycan and O-glycan compositions. (Glyco)peptides were identified based on the accurate mass of the precursor ions with tolerance set at $10 \mathrm{ppm}$ and by matching MS/MS fragmentation with theoretical MS/MS spectra generated from in silico digestion of the provided protein database.

A total of $33 \mathrm{HDL}$-associated proteins were monitored in this study including apolipoprotein A-I (APOA1), apolipoprotein(a) (LPA), apolipoprotein A-II (APOA2), apolipoprotein A-IV (APOA4), apolipoprotein A-V (APOA5), apolipoprotein C-II (APOC2), apolipoprotein C-IV (APOC4), apolipoprotein F (APOF), apolipoprotein L1 (APOL1), haptoglobin-related protein (HPR), phosphatidylcholine-sterol acyltransferase (LCAT), phospholipid transfer protein (PLTP), alpha-1-antitrypsin (A1AT), alpha-1B-glycoprotein (A1BG), alpha-1-antichymotrypsin (AACT), apolipoprotein B-100 (APOB100), apolipoprotein C-I (APOC1), apolipoprotein C-III (APOC3), apolipoprotein D (APOD), apolipoprotein E (APOE), beta-2-glycoprotein $1(\mathrm{APOH})$, apolipoprotein M (APOM), complement C1s subcomponent (C1S), clusterin (CLUS or APOJ), complement C3 (C3), alpha-2-HS-glycoprotein (FETUA or AHSG), hemopexin (HPX), heparin cofactor 2 (HCF2), kininogen-1 (KNG1), serum paraoxonase/arylesterase 1 (PON1), serum amyloid A-4 (SAA4), serum amyloid A-1 (SAA1), serum amyloid A-2 (SAA2). 


\section{Supporting Information Method 2: \\ Targeted glycoproteomics analysis}

Tryptic digestion of the HDL samples was done in a 96-well format to facilitate batch processing. Samples were randomized before plating. All reagents were freshly prepared in a buffer of $50 \mathrm{mM}$ ammonium bicarbonate. A sample volume of $10 \mu \mathrm{L}$ purified HDL was used for tryptic digestion. After every 20 samples, $10 \mu \mathrm{L}$ of commercially available human serum (SigmaAldrich) was also digested to serve as sample preparation controls. Protein standards (APOA1, APOC1, APOD, APOE, CLUS; all from Sigma-Aldrich) were mixed in known amounts (250, 250, 125,200 , and $125 \mu \mathrm{g} / \mathrm{mL}$, respectively) and digested with the batch to serve as calibration standards. Serial dilution of the digested protein mixture provided the calibration curve for absolute quantitation of the 5 proteins. The digested mixture was diluted by factors of 160,80 , $40,20,16,8,4,2$, and 1 to obtain 9 calibration standards, from which calibration curves spanning 4 orders of magnitude were calculated.

After pipetting the samples, controls, and standards onto the 96 -well plate, $10 \mu \mathrm{L}$ of 100 $\mathrm{mM}$ dithiothreitol was added to each well to reduce the protein disulfide bonds. Protein denaturation was continued by heating in a water bath for $1 \mathrm{~h}$ at $65^{\circ} \mathrm{C}$. Samples were then alkylated with $5 \mu \mathrm{L}$ of $360 \mathrm{mM}$ iodoacetamide for $30 \mathrm{~min}$ at room temperature away from light. Excess iodoacetamide was quenched with $5 \mu \mathrm{L}$ of $100 \mathrm{mM}$ dithiothreitol. Proteins were digested with $10 \mu \mathrm{l}$ of $200 \mu \mathrm{g} / \mathrm{mL}$ sequencing grade trypsin for $18 \mathrm{~h}$ at $37^{\circ} \mathrm{C}$. The digestion was stopped by acidifying the solution with $5 \mu \mathrm{L} 10 \%(\mathrm{v} / \mathrm{v})$ formic acid (Fluka). To account for batch variability and possible run-order effects, $5 \mu \mathrm{L}$ of a $1 \mu \mathrm{g} / \mathrm{mL}$ synthetic peptide with sequence RPAIAINNPYVPR (Bionexus, Oakland, CA) was added as an internal standard. The final volume of the HDL digest is $50 \mu \mathrm{L}$, a 5 -fold dilution of the purified HDL solution. The samples were injected into the LC-MS instrument without further cleanup.

(Glyco)peptides were quantified on an Agilent 1290 Infinity II LC system coupled to an Agilent 6495B Triple Quadrupole MS. Injection volumes were set at $5 \mu \mathrm{L}$ for protein standards 
and HDL samples, and $1 \mu \mathrm{L}$ for serum digests. A pooled sample of the digested serum was run after every $10 \mathrm{HDL}$ samples to serve as quality control (QC). They were used to monitor the stability of the instrument and the reproducibility of the batch analysis.

The HPLC was equipped with a 150 mm Agilent Zorbax Eclipse Plus C18 column with 1.8 $\mu \mathrm{m}$ particle size. A C18 column guard was used to protect the column from the buildup of lipids and other hydrophobic substances in the sample. A binary gradient of (A) $3 \%$ acetonitrile with $0.1 \%$ formic acid in water and (B) $90 \%$ acetonitrile with $0.1 \%$ formic acid in water was set at a flow rate of $0.5 \mathrm{~mL} / \mathrm{min}$. The HPLC pump parameters were programmed to ramp from $0 \%$ to $20 \% \mathrm{~B}$ in $20 \mathrm{~min}, 30 \%$ at $40 \mathrm{~min}, 44 \%$ at $47 \mathrm{~min}$, and $100 \%$ at $48 \mathrm{~min}$ followed by $12 \mathrm{~min}$ column flushing cycle with $100 \% \mathrm{~B}$ and 7 min equilibration at $100 \% \mathrm{~A}$. The Electrospray Ionization (ESI) voltage was set to $3500 \mathrm{~V}$ in the positive mode.

A transition list for target analytes was created by combining previously reported transitions $(25,26)$ with new transitions selected from the Orbitrap analysis. The transition list included 47 peptides and 163 glycopeptides from 33 proteins. The instrument was run on Dynamic Multiple Reaction Monitoring (DMRM) mode to minimize the number of transitions being monitored at each scan cycle. For peptides, at least 2 product ions were selected for monitoring. Quantitation was based on the area of the more abundant product ion while the others are for qualitative identification. Product ions for glycopeptides were based on diagnostic glycan fragments. Glycans yield characteristic oxonium ions after Collision Induced Dissociation (CID) with mass-to-charge ratios $(\mathrm{m} / \mathrm{z})$ of $204.08,274.09$, and 366.14 for N-acetylhexosamine (HexNAc), N-acetylneuraminic acid (NeuAc) with loss of $\mathrm{H} 2 \mathrm{O}$, and hexose + HexNAc (Hex1HexNAc1) respectively. 